\title{
PARTITION AS A SOLUTION TO ETHNIC WAR An Empirical Critique of the Theoretical Literature
}

\author{
By NICHOLAS SAMBANIS*
}

\section{Introduction: The Theoretical Case for Partition}

$\mathrm{I}_{\mathrm{p}}^{\mathrm{N}}$ $\mathrm{N}$ two influential articles Chaim Kaufmann elaborated a set of hypotheses on the usefulness of partition as a solution to ethnic civil war, building on the arguments of John Mearsheimer and Stephen Van Evera. ${ }^{1}$ Before them, a first wave of theorists had considered the benefits and costs of partition. A prominent theorist, Donald Horowitz, suggested that

if the short run is so problematical, if the constraints on policy innovation are many, if even grand statements need patchwork readjustment, perhaps it is a mistake to seek accommodation among the antagonists. If it is impossible for groups to live together in a heterogeneous state, perhaps it is better for them to live apart in more than one homogeneous state, even if this necessitates population transfers. Separating the antagonists-partition-is an option increasingly recommended for consideration where groups are territorially concentrated. ${ }^{2}$

It is hard to argue with such a statement. Assuming that "the constraints of policy innovation" and "the short run" can be accurately measured ex ante, it would be easy to recommend partition for some

\footnotetext{
${ }^{*}$ I thank Michael Doyle, Jeff Herbst, Chris Paxson, Russell Leng, George Tsebelis, Philippos Savvides, and three anonymous referees for their very useful comments and suggestions. This article is part of a World Bank project on "The Economics of Political and Criminal Violence." The opinions and any errors in this paper are the author's and they do not necessarily reflect the views of the World Bank, its executive directors, or the countries they represent.

${ }^{1}$ Chaim Kaufmann, "Possible and Impossible Solutions to Ethnic Civil Wars," International Security 20 (Spring 1996); idem, "When All Else Fails," International Security 23 (Fall 1998); John J. Mearsheimer and Stephen Van Evera, "When Peace Means War," New Republic (December 1995).

${ }^{2}$ Donald L. Horowitz, Ethnic Groups in Conflict (Berkeley: University of California Press, 1985), 588. See also Arend Lijphart, Democracy in Plural Societies (New Haven: Yale University Press, 1977), 44-47; Robert A. Dahl, Polyarchy: Participation and Opposition (New Haven: Yale University Press, 1971), 121; and Samuel P. Huntington, "Civil Violence and the Process of Development," Adelphi Paper no. 83 (London: International Institute for Strategic Studies, 1971), 14. Horowitz also discusses dangers of partition (pp. 588-91).
}

World Politics 52 (July 2000), 437-83 
countries while trying to patch up others. However, neither the first nor the second wave of theorists was able to produce operational criteria for applying the theory consistently across cases.

Despite this lack of operational applicability and clarity, partition theory, with its intuitive appeal, has been shaping scholarly and policy opinion on how to end ethnic civil wars. To help policymakers make informed decisions about the usefulness of partition as a strategy to end civil war, I compiled a new data set of all civil wars in the post-World War II era and used that data set to empirically test the set of hypotheses that constitute partition theory.

I focus on the second wave of partition theorists, who have had the greatest impact on the debate. According to them, ethnic violence implies that civil politics cannot be restored unless "ethnic groups are demographically separated into defensible enclaves. ... Solutions that aim at restoring multi-ethnic civil politics and at avoiding population transfers-such as power-sharing, state re-building, or identity reconstruction-cannot work because they do nothing to dampen the security dilemma." 3

The so-called security dilemma lies at the core of partition theory. The dilemma in its purest form arises when one community faces a distrustful other and one's actions to increase one's own security are perceived as threatening the security of others. ${ }^{4}$ Posen argues that this dynamic is intensified when the opponents belong to different ethnic groups. ${ }^{5}$ Ethnic civil wars, argue partition theorists, are characterized by strong and fixed identities, by weak ideological and strong religious overtones, by the dissemination of tales of atrocities to strengthen mobilization, and by easy recognition of identities and the existence of only limited scope for individual choice. Therefore, once war starts, the theory goes, all members of the group must be mobilized because other ethnic groups will inevitably recognize them as enemies. ${ }^{6}$ This inescapable destiny reinforces the dynamics of war and must lead to partition, since "once ethnic groups are mobilized for war, the war cannot end until the populations are separated into defensible, mostly homogeneous regions.... Ethnic separation ... allows ... cleansing and rescue imperatives [to] disappear; war is no longer mandatory."

\footnotetext{
${ }^{3}$ Kaufmann (fn. 1, 1996), 137, 139.

${ }^{4}$ See Robert Jervis, "Cooperation under the Security Dilemma," World Politics 30 (January 1978). Such suspicion and fear would be supported by actual or perceived state collapse, which transforms the domestic political environment into a near anarchic environment.

${ }^{5}$ Barry Posen, "The Security Dilemma and Ethnic Conflict," Survival 35 (Spring 1993).

${ }^{6}$ Kaufmann (fn. 1, 1996), 139-47.

${ }^{7}$ Ibid., 150.
} 
However intuitive that reasoning may be, it is nothing more than a series of unsubstantiated assertions. Beyond a handful of self-selected cases, partition theorists have not presented proof that partition is the only viable and credible solution to ethnic civil war. They have not even proven that partition outperforms other war outcomes in terms of peace-building potential. The theory is indeed plausible under strict assumptions, but are these assumptions realistic?

This paper poses a serious challenge to partition theorists by providing a rigorous test of the theory with a comparison of post-civil war realities in both partitioned and nonpartitioned states. It focuses on countries that have experienced civil war; it does not consider cases of peaceful partition. ${ }^{8}$ I begin by summarizing other authors' critiques of partition theory in the next section. I then identify the main determinants of war-related partition and test the three core hypotheses of partition theory: (1) that partitions facilitate postwar democratization; (2) that they prevent war recurrence; and (3) that they significantly reduce residual low-level ethnic violence. My tests lead me to reject the most critical tenets of partition theory. I find that partitions do not help prevent recurrence of ethnic war and that they may not even be necessary to stop low-level ethnic violence. Although it may seem like a clean and easy solution, partition fares no better than other outcomes of ethnic civil war. I turn now to a summary of the debate.

\section{REBUTTALS AND COUNTERREBUTTALS: The STATE of the Debate on Partition}

The suggestion that populations must be forcibly separated to prevent them from killing each other has inspired both approval and criticism. The most significant criticism is that partition may be too limiting a

\footnotetext{
${ }^{8}$ Partition theorists also approach the problem in this way: they do not discuss partition as a preventive measure before war occurs but rather analyze it as a strategy to end civil war after it occurs"when all else fails," as Kaufman (fn. 1, 1998) puts it. Peaceful partitions therefore cannot offer any information on my main research question-war recurrence-since a war is a necessary precondition for war recurrence. My research design is therefore the equivalent of a biostatistician's inquiry into the effects of medical treatment for illness: suffering from that illness is a precondition for inclusion in the study. Studying the relationship between initial war occurrence and partition would be an interesting extension of my study. The research question would have to be reformulated, as would the data set. The dependent variable could no longer be war recurrence or residual violence and one would need a theory of civil war occurrence that included partition as a potentially important determinant of civil war (or civil peace). Such a study would analyze a random sample of countries (or the entire population of countries) and would have to include both countries that experienced war and countries that were at peace. To identify whether partition causes war, one could code a binary variable denoting if the country was partitioned and use it as a regressor in a model of the onset of war. Alternatively, one could estimate two separate regressions on partitioned and nonpartitioned countries and compare the coefficients. In terms of the medical research example above, this study would effectively ask: how does factor $x$ affect one's chances of becoming ill?
} 
solution and that ethnic cooperation may be possible even after civil war, facilitated by both ethnic diffusion and third-party security guarantees. ${ }^{9}$ Some say that partition is also too severe a solution, as forced population movements cause tremendous human suffering and violate important human rights. ${ }^{10}$ The process of partition may also create undemocratic successor states, which would be likely to repress their residual minorities much as their predecessors did. ${ }^{11}$ This is important because successor states will rarely be ethnically homogeneous and may incorporate new ethnic antagonisms. ${ }^{12}$ Moreover, partition does not resolve underlying ethnic rivalry, so civil wars that end in partition could be transformed into interstate wars between predecessor and successor states. ${ }^{13}$ Finally, endorsing some partitions may encourage partition movements elsewhere, leading to new wars. ${ }^{14}$

The debate between partition theorists and their critics is ongoing, although some of the critiques listed above have been settled or are close to being settled in the literature. Below, I summarize the status of three important arguments.

\section{Successful Ethnic Partitions Do Not Encourage Partition MOVEMENTS ELSEWHERE}

Critics have argued that support by the international community for partition in a few countries would encourage partitions elsewhere. Kaufman, however, has rebutted this criticism by arguing that the uncertainty and extreme costs of civil war would discourage the initiation of partition movements unless such movements are inevitable for domestic political reasons. ${ }^{15}$ That position is partially supported by a set of analyses of the "international spread of ethnic conflict" which suggests that cross-country contagion effects of ethnic partition movements are rare. ${ }^{16}$

\footnotetext{
${ }^{9}$ On ethnic diffusion cooperation, see Daniel L. Byman, "Divided They Stand: Lessons about Partition from Iraq and Lebanon," Security Studies 7 (Autumn 1997). On security guarantees and ethnic war termination, see Barbara F. Walter, "The Critical Barrier to Civil War Settlement," International Organization 51 (Summer 1997). Neither Byman nor Walter is a critic of partition theory (Byman in fact supports partition under certain conditions). Some of their arguments, however, can be read as indirect critiques of the theory.

${ }^{10}$ Radha Kumar, "The Troubled History of Partition," Foreign Affairs 76 (January-February 1997).

${ }^{11}$ Ibid; see also Amitai Etzioni, "The Evils of Self-Determination," Foreign Policy 89 (Winter 1992-93); and Robert Schaeffer, Warpaths: The Politics of Partition (New York: Hill and Wang, 1990).

12 Byman (fn. 9).

13 Ibid.; and Schaeffer (fn. 11).

${ }^{14}$ Etzioni (fn. 11); Allen Buchanan "Self-Determination and the Right to Secede," Journal of International Affairs 45 (Winter 1992).

${ }^{15}$ Kaufman (fn. 1, 1998).

${ }^{16}$ David A. Lake and Donald Rothchild, eds., The International Spread of Etbnic Conflicts (Princeton: Princeton University Press, 1998). More research is needed to fully appreciate the impact of historical examples and of precedential reasoning in ethnic conflict.
} 


\title{
Successor States May Also Incorporate
}

\author{
ETHNIC CONFLICT
}

According to partition theorists, the success of partition depends on the demographic reorganization of the new territories and on the absence of militarily significant minorities in the new states. However, successor states in most actual cases of partition are not ethnically pure. Hence, this core premise of partition theory may be unrealistic. ${ }^{17}$ To quote Horowitz:

The linchpin of all the arguments [for partition] is the assumption that the probable outcome of secession and partition will be more homogeneous states and, concomitantly, a lower ethnic conflict level. If the assumption were correct, the conclusion would follow. But the assumption is wrong: the only thing secession and partition are unlikely to produce is ethnically homogeneous or harmonious states. ${ }^{18}$

Furthermore, even if successor states were homogeneous, the mobilization perspective of ethnic conflict would suggest that, unless partition is accompanied by regime or leadership reform, there is no guarantee that ethnic groups in successor states will not be mobilized into another war against residual minorities. ${ }^{19}$ So again the theory's claims depend critically on unrealistic premises about the ethnic composition and political institutions of successor and predecessor states.

This last point is related to the theory's dependence on the concept of the security dilemma, which ignores the fact that conflict is often due not to the defensive security needs of ethnic groups but rather to the "predatory" goals of their leaders. It is worth noting that even the "father" of the concept of the security dilemma-Robert Jervis-has acknowledged that in most contemporary civil conflicts there are not only security motives but also predatory ones. It follows that partition will not resolve the security dilemma of the partitioned ethnic groups if it exacerbates the "predatory" incentives of predecessor states. ${ }^{20} \mathrm{Yu}$ goslavia's recurrent wars are a case in point.

${ }^{17}$ See Horowitz (fn. 2), 588-91 and chaps. 2, 6.

${ }^{18}$ Ibid., 589, emphasis added.

${ }^{19}$ See, among others, David A. Lake and Donald Rothchild, "Containing Fear: The Origins and Management of Ethnic Conflict," International Security 21 (Fall 1996); V. P. Gagnon, "Ethnic Nationalism and International Conflict: The Case of Serbia," International Security 19 (Winter 1995); Rui J. P. de Figueiredo and Barry R. Weingast, "The Rationality of Fear: Political Opportunism and Ethnic Conflict," in Barbara Walter and Jack Snyder, eds., Civil Wars, Insecurity, and Intervention (New York: Columbia University Press, 1999).

${ }^{20}$ David Laitin, "Somalia: Civil War and International Intervention," in Walter and Snyder (fn. 19); and Jack Snyder and Robert Jervis "Civil War and the Security Dilemma," in Walter and Snyder (fn. 19), 19-24. 


\section{Ethnic Cooperation Is Possible without Partition}

There can be many ways to resolve the security dilemma. ${ }^{21}$ Noncooperative game theory identifies a number of conditions under which a mutually beneficial Nash equilibrium can be achieved between parties whose preferences are, first, to cheat their opponent into cooperating while they defect and, second, to mutually cooperate rather than mutually defect-preferences such as those characterizing many ethnic conflicts. Perspectives on international negotiation have also suggested that the parties can cooperate if they negotiate a solution to "delegate to neutral authorities." 22 If negotiation is a viable option, it may be possible to reach an internationally or regionally brokered agreement that addresses the conflict's underlying causes. ${ }^{23}$

The problem with these solutions is that they may not be credible, which reinforces the security dilemma. ${ }^{24}$ Thus, argues Walter, civil wars tend not to end in negotiated settlements, and a settlement will hold only when external security guarantees are available. ${ }^{25}$ However, it need not follow that all peace agreements and institutional solutions to ethnic conflict are noncredible. Only the warring parties can gauge ex ante whether an institutional framework designed to end the war will be successful because they know their opponents and can estimate the probability that the peace process will fail. ${ }^{26}$ Also, partition, which is allegedly credible because it redraws national borders to resolve the minority's security dilemma, is as vulnerable to the credibility argument as any other solution, since only robust external security guarantees can credibly prevent predatory predecessor states from restarting the war against successor states.

What if there is no decisive end to the war (such as a military victory) and ethnic competition persists, threatening the possibility of new violence? The rationalist perspective on war would suggest that the war should have resolved any uncertainty about relative resolve and power that might have led to war in the first place. Thus, miscalculations

${ }^{21}$ I do not develop a theory of ethnic cooperation in this paper. I only summarize relevant theoretical arguments to frame my empirical analysis. Thus, this section is not designed to resolve all doubt about the possibility of ethnic cooperation after civil war.

${ }^{22}$ Snyder and Jervis (fn. 20), 18. On power sharing, see Timothy Sisk, Power Sharing and International Mediation in Ethnic Conflicts (Washington, D.C: United States Institute of Peace, 1996).

${ }^{23}$ Lake and Rothschild (fn. 19).

${ }^{24}$ Snyder and Jervis ( $\left.\mathrm{fn} .20\right)$.

${ }^{25}$ In Walter's (fn. 9) argument, the security dilemma depends on an asymmetry of power between the government and rebels. Walter notes that credible external security guarantees are effective, though difficult. The difficulty in proving the credibility of the third party's commitment amounts to indirect support for the partition thesis, though only if partition is proven to be more credible and less difficult to implement than a brokered settlement.

${ }^{26}$ De Figuereido and Weingast (fn. 19). 
would be less likely after the first war and rational parties would prefer not to start a new war regardless of the first war's outcome. ${ }^{27}$ This could change, however, as the parties' relative capabilities change over time. So one way for the international community to enable stable peace is to preserve the military balance that follows the end of the war. An alternative, which could work better in some situations, is to create a regional hegemon responsible for regional peace. ${ }^{28}$

Finally, cooperation among ethnic groups may be possible if ethnic diffusion increases as a result of the war, that is, if the opposite of partition takes place. Byman has suggested that increased ethnic diffusion may mitigate the security dilemma, since it would reduce the probability that a single ethnic group could become politically and militarily dominant. His argument derives from the theoretical literature on international alliances and posits that ethnic "balancing" against threatening groups is both possible and stabilizing. ${ }^{29}$ This hypothesis has yet to be tested, but it is relevant to note that a budding political economy literature on civil wars has identified a parabolic relationship between ethnic fragmentation and the probability of civil war; that is, the probability of civil war drops significantly at very high levels of ethnic diversity and it is greatest in ethnically polarized societies, which seems to support Byman's hypothesis. ${ }^{30}$ The question that partition theorists raise is slightly different, however: can ethnic diversity reduce the risk of war recurrence after the first war ends? I answer this question in later sections.

\section{TAKING Sides: NeW DaTA AND NeW EMPIRICAL TESTS OF PARTITION THEORY}

The four most important questions in partition theory are still unresolved. (1) What are the main determinants of partition? (2) Does par-

${ }^{27}$ The rationalist school is well represented by Geoffrey Blainey, The Causes of War (New York: Free Press, 1973); and James Fearon, "Rationalist Explanations for War," International Organization 49 (Summer 1995). War should reveal any private information about relative power and resolve, making it less rational for parties to resort to war again rather than to strike a more efficient bargain short of war. That said, we should also consider other explanations of war and weigh them against this argument.

${ }_{28}^{28}$ Snyder and Jervis (fn. 20); and Laitin (fn. 20).

${ }^{29}$ Byman (fn. 9). This argument can backfire. Ethnic balancing can also paralyze the state. For such an argument, see Harrison Wagner, "The Causes of Peace," in Roy Licklider, ed., Stopping the Killing (New York: New York University Press, 1993). Wagner argues that because military victory results in unitary political systems, it will be more stable than any peace agreement based on ethnic balancing. Indeed, the occurrence of an ethnic war suggests a precedent of failed ethnic balancing. In this paper, I present empirical results about the relationship of ethnicity to postwar violence, but that relationship also demands better theorizing.

${ }^{30}$ See, e.g., Paul Collier, Ibrahim Elbadawi, and Nicholas Sambanis, "How Much War Will We See? Estimating the Probability of Civil War in 161 Countries" (Manuscript, World Bank, February 2000). 
tition create democratic or undemocratic states? (3) Does partition prevent war recurrence? And (4) does partition end low-level ethnic violence (that is, violence short of war)?

To answer these questions, I have compiled a new cross-sectional data set of all civil wars since 1944. The unit of observation is a civil war. The analysis focuses on wars that have been over for at least two years at the time of writing, but also included are eight ongoing wars to capture the partition theorists' interest in partition as a way of ending ongoing wars. ${ }^{31}$

A civil war is defined as an armed conflict that has (1) caused more than one thousand deaths; (2) challenged the sovereignty of an internationally recognized state; (3) occurred within the recognized boundaries of that state; (4) involved the state as one of the principal combatants; (5) included rebels with the ability to mount an organized opposition; and (6) involved parties concerned with the prospect of living together in the same political unit after the end of the war. ${ }^{32}$ This definition allows me to combine wars from several data sets. ${ }^{33}$ Detailed

${ }^{31}$ Dropping those cases did not affect any of the results presented in later sections.

${ }^{32}$ This definition is nearly identical to the definition of a civil war in J. David Singer and Melvin Small, Correlates of War Project: International and Civil War Data, 1816-1992 (Ann Arbor, Mich.: ICPSR, 1994); idem, Resort to Arms (Beverly Hills, Calif.: Sage Publications, 1982); and Roy Licklider, "The Consequences of Negotiated Settlements in Civil Wars, 1945-1993," American Political Science Review 89 (September 1995). Unlike them, my coding of wars does not presume one thousand deaths per year, but rather uses the one thousand deaths as the threshold for the entire war. In fact, however, most of my cases have caused one thousand deaths annually. My coding decision was based on the arbitrariness of setting one thousand as the annual death criterion and on the lack of available data on annual deaths in the Correlates of War project. Indeed, the codebook of the ICPSR study, which includes the international and civil war data files for the Correlates of War Project, does not mention an annual death threshold and no annual death data are made available by the authors.

${ }^{33}$ My sources for coding wars include Singer and Small (fn. 32, 1994); Licklider (fn. 32); idem (fn. 29); Peter Wallensteen and Margareta Sollenberg, "Armed Conflicts, Conflict Termination, and Peace Agreements, 1989-1996," Journal of Peace Research 34, no. 3 (1997); Daniel C. Esty et al., "The State Failure Project: Early Warning Research for US Foreign Policy Planning," in John L. Davies and Ted Robert Gurr, eds., Preventive Measures: Building Risk Assessment and Crisis Early Warning Systems (Boulder, Colo., and Totowa, N.J.: Rowman and Littlefield, 1998); David Mason and Patrick Fett, "How Civil Wars End: A Rational Choice Approach," Journal of Confict Resolution 40 (December 1996); Patrick Regan, "Conditions for Successful Third Party Interventions," Journal of Conflict Resolution 40, no. 1 (1996); Walter (fn. 9); SIPRI, SIPRI Yearbook (http://editors.sipri.se/pubs/yearb.html); Human Rights Watch, World Report (New York and Washington, D.C.: Human Rights Watch, various years). Secondary texts consulted include Robert I. Rotberg, ed., Burma: Prospects for a Democratic Future (Washington, D.C.: Brookings Institution, 1998); Martin Stuart-Fox, A History of Laos (Cambridge: Cambridge University Press, 1998); David Callahan, Unwinnable Wars (New York: Hill and Wang, 1997); John O. Iatrides, "The Doomed Revolution: Communist Insurgency in Postwar Greece," in Licklider (fn. 29); Michael W. Doyle, Robert Orr, and Ian Johnstone, eds., Keeping the Peace (Cambridge: Cambridge University Press, 1997); Francis M. Deng, War of Visions: Confict of Identities in the Sudan (Washington, D.C.: Brookings Institution, 1999); David McDowall, A Modern History of the Kurds (New York: St. Martin's Press, 1996); and Nicholas Sambanis, "United Nations Peacekeeping in Theory and in Cyprus: New Conceptual Approaches and Interpretations" (Ph.D. diss., Princeton University, 1999). The most important difference between my coding and that of others concerns the periodization of wars. I have broken what is a single observation of war in other data 
documentation on my coding and sources for all the variables in the data set is available online. ${ }^{34}$

\section{Definition of Partition}

The variable denoting partition, PART, is binary and equals 1 if an event of partition is observed and 0 otherwise. Partition is defined as a war outcome that involves both border adjustment and demographic changes. This is a broad definition that differs slightly from Kaufmann's. To justify a narrower definition of partition, Kaufmann wrote that "we should focus on partition rather than secession . . . to assess whether international intervention reduces or increases the costs of ethnic conflict." $35 \mathrm{He}$ then defined partitions as "separations jointly decided upon by the responsible powers: either agreed between the two sides (and not under pressure of imminent military victory by one side), or imposed on both sides by a stronger third party ... [and he defined] secessions as new states created by the unilateral action of a rebellious ethnic group." ${ }^{36}$

I do not find the narrow definition convincing or useful, given that the far-reaching implications of partition theory affect secessions and partitions equally in the minds of most policymakers and academics. Moreover, the narrow definition reclassifies as secessions certain cases that Kaufmann originally treated as partitions. ${ }^{37}$ Finally, this definition does not justify the inclusion of some of the partitions included on Kaufmann's own list. ${ }^{38}$ An example is Cyprus, which Kaufmann correctly-though for the wrong reasons-classifies as a de facto partition. ${ }^{39}$ The 1974 partition of Cyprus was neither the outcome of an agreement nor an imposition by a third party, as the narrow definition would have it. Rather, it was the result of military victory by the Turkish side. ${ }^{40}$

Given these problems with the narrow definition, I use the broader definition, combining cases of partition and secession listed in Kauf-

sets into more than one observation; or, conversely, I have collapsed two or more observations in one by uniformly applying this rule: a war is coded as a single observation if the parties and issues are the same, if the war events are not separated by a substantial period of nonviolence, and/or if the parties sign a peace agreement or agree to a major truce.

${ }_{34}$ The document can be downloaded from http://www.worldbank.org/research/conflict/data.htm.

${ }^{35}$ Kaufmann (fn. 1, 1998), 125.

${ }^{36}$ See Kaufmann (fn. 1, 1998), 125, fn. 21.

${ }^{37}$ Kaufmann (fn. 1, 1996).

${ }^{38}$ Kauffman (fn. 1, 1998).

39 Ibid.

${ }^{40}$ It is well known to scholars of the Cyprus problem that Turkey and the Turkish Cypriots constituted and acted as a single party both during the violent part of that conflict (1963-74) and during the subsequent negotiation phases; see Sambanis (fn. 33). 
mann's two articles. ${ }^{41} \mathrm{I}$ also add cases that satisfy my definition but are not on Kaufmann's list. ${ }^{42}$ Table 1 lists all civil wars and partitions in my data set, sorted by country name, war start/end dates, the type of war, war recurrence, and lower-level violence outcomes. ${ }^{43}$

In my analysis of democratization, war termination, and low-level political violence I use explanatory variables that other authors have identified as significant for those events. These variables are important both for the theory of partition and for use as controls in subsequent empirical tests. Table 2 presents summary statistics for all variables and explains what each one measures. Table 3 presents a correlation matrix with the most important variables used in the analysis.

I now turn to the question of the determinants of partition.

\section{Main Determinants of Partition}

My data set includes 125 civil wars, which produced 21 partitions. ${ }^{44}$ Using the entire data set (which includes six right-truncated wars), I estimated probit models of the incidence of partition, selecting the explanatory variables on the basis of theory developed in the literature on civil war. ${ }^{45}$ I want to test whether some of the same variables that either cause or terminate civil wars are also significant determinants of warrelated partition.

I make the following testable hypotheses: Following the reasoning of partition theorists, I hypothesize that WARTYPE (ethnic/religious rather than ideological war) should be positively associated with parti-

\footnotetext{
${ }^{41}$ Kaufmann (fn.1, 1996 and 1998). Other studies also use the broad definition, given that the distinction between secession and partition seems artificial. See, among others, Horowitz (fn. 2); and Alexis Heraclides, The Self-Determination of Minorities in International Politics (Portland: Frank Cass, 1991); both use the terms partition and secession interchangeably.

${ }^{42}$ I consider only post-World War II cases because of the paucity of economic data from before 1945. Thus, I exclude the partition of Ireland. Cases of peaceful partition are also excluded, for example, Macedonia (1992), Czechoslovakia (1993), and Singapore (1965). I exclude one case (Iraq) that I believe was erroneously classified as a partition in Kaufmann (fn. 1, 1998). I exclude Iraq (1991) because there is no recognized, functional, or even autonomous Iraqi Kurdistan and the territory and its population would have been within reach of the Iraqi military had it not been for the U.S.-enforced no-fly zone.

${ }^{43} \mathrm{My}$ coding of cases of partition incorporated suggestions made by anonymous referees.

${ }^{44}$ One might argue that the "real" number of partitions is smaller, since several of them occurred in either the former Yugoslavia or the former USSR. This would imply that these partitions may not be independent of one another. Thus, I cluster all same-country observations in my statistical analysis, relaxing the assumption of independence for those observations and allowing for nonconstant variance within clusters.

${ }^{45}$ See, for example, Paul Collier and Anke Hoeffler, "Justice-Seeking and Loot-Seeking in Civil War" (Manuscript, World Bank, February 1999); Michael W. Doyle and Nicholas Sambanis, "International Peacebuilding: A Theoretical and Quantitative Analysis" (Manuscript, Princeton University and the World Bank, February 2000); Paul Collier, "On the Economic Consequences of Civil War," Oxford Economic Papers 51 (1998); and Mason and Fett (fn. 33).
} 


\begin{tabular}{|c|c|c|c|c|c|c|}
\hline $\begin{array}{l}\text { Country Name } \\
\text { Where Civil War } \\
\text { Took Place }\end{array}$ & $\begin{array}{c}\text { Year } \\
\text { War } \\
\text { Started }\end{array}$ & $\begin{array}{l}\text { Year } \\
\text { War } \\
\text { Ended }\end{array}$ & $\begin{array}{l}\text { Did War } \\
\text { End for } \\
2 \text { years? }\end{array}$ & $\begin{array}{c}\text { Did } \\
\text { Residual } \\
\text { Violence } \\
\text { End for } \\
2 \text { years? }\end{array}$ & $\begin{array}{c}\text { Was } \\
\text { There a } \\
\text { Partition? }\end{array}$ & $\begin{array}{l}\text { Type of War } \\
\text { (Identity } \\
\text { or Not?) }\end{array}$ \\
\hline Afghanistan & 1978 & 1992 & no & no & no & ideology/other \\
\hline Afghanistan & 1993 & ongoing & no & no & no & ethnic/religious \\
\hline Algeria & 1962 & 1963 & yes & yes & no & ideology/other \\
\hline Algeria & 1992 & 1997 & no & no & no & ethnic/religious \\
\hline Angola & 1975 & 1991 & no & no & no & ethnic/religious \\
\hline Angola & 1992 & ongoing & no & no & no & ethnic/religious \\
\hline Argentina & 1955 & 1955 & yes & yes & no & ideology/other \\
\hline Azerbaijan & 1988 & 1996 & yes & yes & yes & ethnic/religious \\
\hline Bangladesh & 1973 & 1994 & yes & yes & no & ethnic/religious \\
\hline Bolivia & 1952 & 1952 & yes & yes & no & ideology/other \\
\hline Burma & 1948 & 1951 & yes & no & no & ideology/other \\
\hline Burma & 1968 & 1982 & no & no & no & ethnic/religious \\
\hline Burma & 1983 & 1995 & yes & no & no & ethnic/religious \\
\hline Burundi & 1965 & 1969 & yes & no & no & ethnic/religious \\
\hline Burundi & 1972 & 1973 & yes & yes & no & ethnic/religious \\
\hline Burundi & 1988 & 1988 & no & no & no & ethnic/religious \\
\hline Burundi & 1991 & ongoing & no & no & no & ethnic/religious \\
\hline Cambodia & 1970 & $1975^{\circ}$ & yes & no & no & ideology/other \\
\hline Cambodia & 1979 & 1991 & yes & yes & no & ideology/other \\
\hline $\begin{array}{l}\text { Central African . } \\
\text { Rep }\end{array}$ & 1995 & 1997 & yes & yes & no & ideology/other \\
\hline Chad & 1965 & 1979 & no & no & no & ethnic/religious \\
\hline Chad & 1980 & 1994 & yes & yes & no & ethnic/religious \\
\hline China & 1967 & 1968 & yes & no & no & ethnic/religious \\
\hline China-Taiwan & 1947 & 1947 & yes & no & yes & ideology/other \\
\hline China-Tibet & 1950 & 1951 & yes & no & no & ethnic/religious \\
\hline Colombia & 1948 & 1962 & yes & yes & no & ideology/other \\
\hline Colombia & 1978 & ongoing & no & no & no & ideology/other \\
\hline Congo Brazzaville & 1992 & 1996 & no & no & no & ideology/other \\
\hline Congo/Zaire & 1967 & 1967 & yes & yes & no & ethnic/religious \\
\hline Congo/Zaire & 1975 & 1979 & yes & no & no & ethnic/religious \\
\hline Congo/Zaire & 1960 & 1965 & no & no & no & ethnic/religious \\
\hline Congo/Zaire & 1996 & 1997 & no & no & no & ethnic/religious \\
\hline Costa Rica & 1948 & 1948 & yes & yes & no & ideology/other \\
\hline Cuba & 1958 & 1959 & yes & no & no & ideology/other \\
\hline Cyprus & 1963 & 1964 & no & no & yes & ethnic/religious \\
\hline Cyprus & 1974 & 1974 & yes & yes & yes & ethnic/religious \\
\hline Djibouti & 1991 & 1995 & yes & yes & no & ideology/other \\
\hline Dominican Rep. & 1965 & 1965 & yes & yes & no & ideology/other \\
\hline El Salvador & 1979 & 1992 & yes & yes & no & ideology/other \\
\hline Ethiopia/Eritrea & 1974 & 1991 & yes & yes & yes & ethnic/religious \\
\hline Ethiopia & 1977 & 1985 & yes & no & no & ethnic/religious \\
\hline Ethiopia & 1974 & 1991 & yes & yes & no & ideology/other \\
\hline
\end{tabular}




\begin{tabular}{|c|c|c|c|c|c|c|}
\hline $\begin{array}{l}\text { Country Name } \\
\text { Where Civil War } \\
\text { Took Place }\end{array}$ & $\begin{array}{c}\text { Year } \\
\text { War } \\
\text { Started }\end{array}$ & $\begin{array}{l}\text { Year } \\
\text { War } \\
\text { Ended }\end{array}$ & $\begin{array}{l}\text { Did War } \\
\text { End for } \\
2 \text { years? }\end{array}$ & $\begin{array}{l}\quad \text { Did } \\
\text { Residual } \\
\text { Violence } \\
\text { Endfor } \\
2 \text { years? }\end{array}$ & $\begin{array}{c}\text { Was } \\
\text { There a } \\
\text { Partition? }\end{array}$ & $\begin{array}{l}\text { Type of War } \\
\text { (Identity } \\
\text { or Not?) }\end{array}$ \\
\hline Georgia & 1991 & 1993 & yes & yes & yes & ethnic/religious \\
\hline Georgia & 1992 & 1994 & yes & yes. & yes & ethnic/religious \\
\hline Greece & 1944 & 1949 & yes & yes & no & ideology/other \\
\hline Guatemala & 1954 & 1954 & yes & yes & no & ideology/other \\
\hline Guatemala & 1966 & 1972 & no & no & no & ethnic/religious \\
\hline Guatemala & 1974 & 1994 & yes & yes & no & ethnic/religious \\
\hline Haiti & 1991 & 1994 & no & no & no & ideology/other \\
\hline Haiti & 1995 & 1996 & yes & yes & no & ideology/other \\
\hline India & 1946 & 1948 & yes & yes & yes & ethnic/religious \\
\hline India & 1965 & 1965 & yes & no & yes & ethnic/religious \\
\hline India & 1984 & 1994 & yes & yes & no & ethnic/religious \\
\hline India & 1989 & 1994 & yes & no & yes & ethnic/religious \\
\hline Indonesia & 1956 & 1960 & yes & no & no & ideology/other \\
\hline Indonesia & 1986 & 1986 & yes & yes & no & ethnic/religious \\
\hline Indonesia & 1950 & 1950 & no & no & no & ethnic/religious \\
\hline Indonesia & 1953 & 1953 & no & no & no & ethnic/religious \\
\hline Indonesia & 1975 & 1982 & yes & no & no & ethnic/religious \\
\hline Iran & 1978 & 1979 & no & no & no & ideology/other \\
\hline Iran & 1981 & 1982 & yes & no & no & ethnic/religious \\
\hline Iraq & 1959 & 1959 & no & no & no & ethnic/religious \\
\hline Iraq & 1961 & 1975 & yes & no & no & ethnic/religious \\
\hline Iraq & 1988 & 1994 & yes & no & no & ethnic/religious \\
\hline Iraq & 1991 & 1994 & yes & no & no & ethnic/religious \\
\hline Israel/Palestine & 1947 & 1949 & no & no & yes & ethnic/religious \\
\hline Israel & 1950 & 1994 & yes & yes & no & ethnic/religious \\
\hline Jordan & 1971 & 1971 & yes & yes & no & ethnic/religious \\
\hline Kenya & 1991 & 1993 & yes & no & no & ethnic/religious \\
\hline Korea & 1950 & 1953 & yes & yes & yes & ideology/other \\
\hline Laos & 1960 & 1975 & yes & no & no & ideology/other \\
\hline Lebanon & 1958 & 1958 & yes & yes & no & ethnic/religious \\
\hline Lebanon & 1975 & 1978 & yes & no & no & ethnic/religious \\
\hline Lebanon & 1982 & 1992 & yes & no & no & ethnic/religious \\
\hline Liberia & 1989 & 1992 & no & no & no & ideology/other \\
\hline Liberia & 1993 & 1996 & yes & no & no & ideology/other \\
\hline Malaysia & 1948 & 1959 & yes & yes & no & ideology/other \\
\hline Mali & 1990 & 1995 & yes & yes & no & ethnic/religious \\
\hline Mexico & 1992 & 1994 & yes & yes & no & ethnic/religious \\
\hline Moldova & 1992 & 1994 & yes & no & yes & ethnic/religious \\
\hline $\begin{array}{l}\text { Morocco/ } \\
\text { W. Sahara }\end{array}$ & 1975 & 1989 & yes & yes & no & ethnic/religious \\
\hline Mozambique & 1979 & 1992 & yes & yes & no & ideology/other \\
\hline Namibia & 1965 & 1989 & yes & yes & no & ethnic/religious \\
\hline Nicaragua & 1978 & 1979 & no & no & no & ideology/other \\
\hline Nicaragua & 1981 & 1989 & yes & yes & no & ideology/other \\
\hline
\end{tabular}




\begin{tabular}{|c|c|c|c|c|c|c|}
\hline $\begin{array}{l}\text { Country Name } \\
\text { Where Civil War } \\
\text { Took Place }\end{array}$ & $\begin{array}{c}\text { Year } \\
\text { War } \\
\text { Started }\end{array}$ & $\begin{array}{c}\text { Year } \\
\text { War } \\
\text { Ended }\end{array}$ & $\begin{array}{l}\text { Did War } \\
\text { End for } \\
2 \text { years? }\end{array}$ & $\begin{array}{c}\text { Did } \\
\text { Residual } \\
\text { Violence } \\
\text { End for } \\
2 \text { years? }\end{array}$ & $\begin{array}{c}\text { Was } \\
\text { There a } \\
\text { Partition? }\end{array}$ & $\begin{array}{c}\text { Type of War } \\
\text { (Identity } \\
\text { or Not?) }\end{array}$ \\
\hline Nigeria & 1967 & 1970 & yes & yes & no & ethnic/religious \\
\hline Nigeria & 1980 & 1984 & yes & no & no & ethnic/religious \\
\hline Northern Ireland & 1968 & 1994 & yes & yes & no & ethnic/religious \\
\hline Pakistan & 1971 & 1971 & yes & yes & yes & ethnic/religious \\
\hline Pakistan & 1973 & 1977 & yes & no & no & ethnic/religious \\
\hline $\begin{array}{l}\text { Papua } \\
\text { New Guinea }\end{array}$ & 1988 & 1991 & yes & no & no & ethnic/religious \\
\hline Paraguay & 1947 & 1947 & yes & yes & no & ethnic/religious \\
\hline Peru & 1980 & 1996 & yes & no & no & ideology/other \\
\hline Philippines & 1950 & 1952 & yes & yes & no & ideology/other \\
\hline Philippines & 1972 & 1996 & yes & no & no & ethnic/religious \\
\hline Philippines & 1972 & 1992 & yes & no & no & ideology/other \\
\hline Romania & 1989 & 1989 & yes & yes & no & ideology/other \\
\hline Russia/Chechnya & 1994 & 1996 & no & no & yes & ethnic/religious \\
\hline Rwanda & 1963 & 1964 & yes & no & no & ethnic/religious \\
\hline Rwanda & 1990 & 1994 & yes & yes & no & ethnic/religious \\
\hline Sierra Leone & 1991 & 1996 & no & no & no & ideology/other \\
\hline Somalia & 1988 & 1991 & no & no & yes & ethnic/religious \\
\hline Somalia & 1992 & ongoing & no & no & no & ethnic/religious \\
\hline South Africa & 1976 & 1994 & yes & yes & no & ethnic/religious \\
\hline Sri Lanka & 1971 & 1971 & yes & yes & no & ideology/other \\
\hline Sri Lanka & 1987 & 1989 & yes & yes & no & ideology/other \\
\hline Sri Lanka & 1983 & ongoing & no & no & no & ethnic/religious \\
\hline Sudan & 1963 & 1972 & yes & yes & no & ethnic/religious \\
\hline Sudan & 1983 & ongoing & no & no & no & ethnic/religious \\
\hline Tajikistan & 1992 & 1994 & yes & no & yes & ethnic/religious \\
\hline Thailand & 1967 & 1985 & yes & yes & no & ethnic/religious \\
\hline Turkey & 1984 & ongoing & no & no & no & ethnic/religious \\
\hline Uganda & 1966 & $1966^{\circ}$ & yes & yes & no & ethnic/religious \\
\hline Uganda & 1978 & 1979 & no & no & no & ideology/other \\
\hline Uganda & 1980 & 1986 & yes & no & no & ethnic/religious \\
\hline Vietnam Rep. & 1960 & 1975 & yes & yes & yes & ideology/other \\
\hline Yemen & 1948 & 1948 & yes & yes & no & ideology/other \\
\hline Yemen & 1994 & 1994 & yes & yes & no & ideology/other \\
\hline Yemen, North & 1962 & 1969 & yes & yes & no & ideology/other \\
\hline Yemen, South & 1986 & 1987 & yes & yes & no & ideology/other \\
\hline Yugoslavia/Bosnia & 1992 & 1995 & yes & yes & yes & ethnic/religious \\
\hline Yugoslavia/Croatia & 1991 & 1991 & no & no & yes & ethnic/religious \\
\hline Yugoslavia/Croatia & 1995 & 1995 & yes & yes & yes & ethnic/religious \\
\hline $\begin{array}{l}\text { Zimbabwe/ } \\
\text { Rhodesia }\end{array}$ & 1972 & 1980 & yes & no & no & ethnic/religious \\
\hline Zimbabwe & 1984 & 1984 & yes & yes & no & ethnid/religious \\
\hline
\end{tabular}




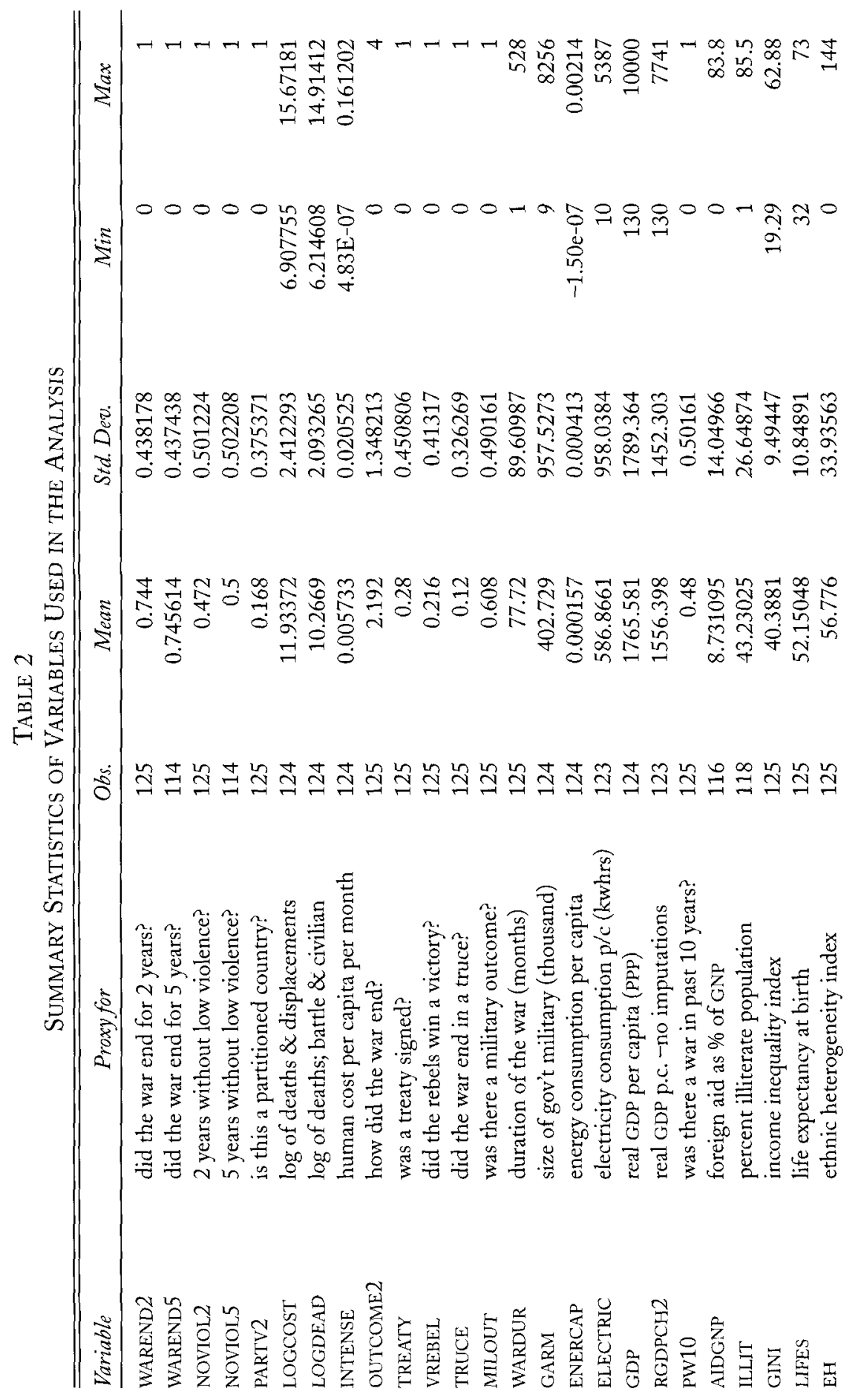




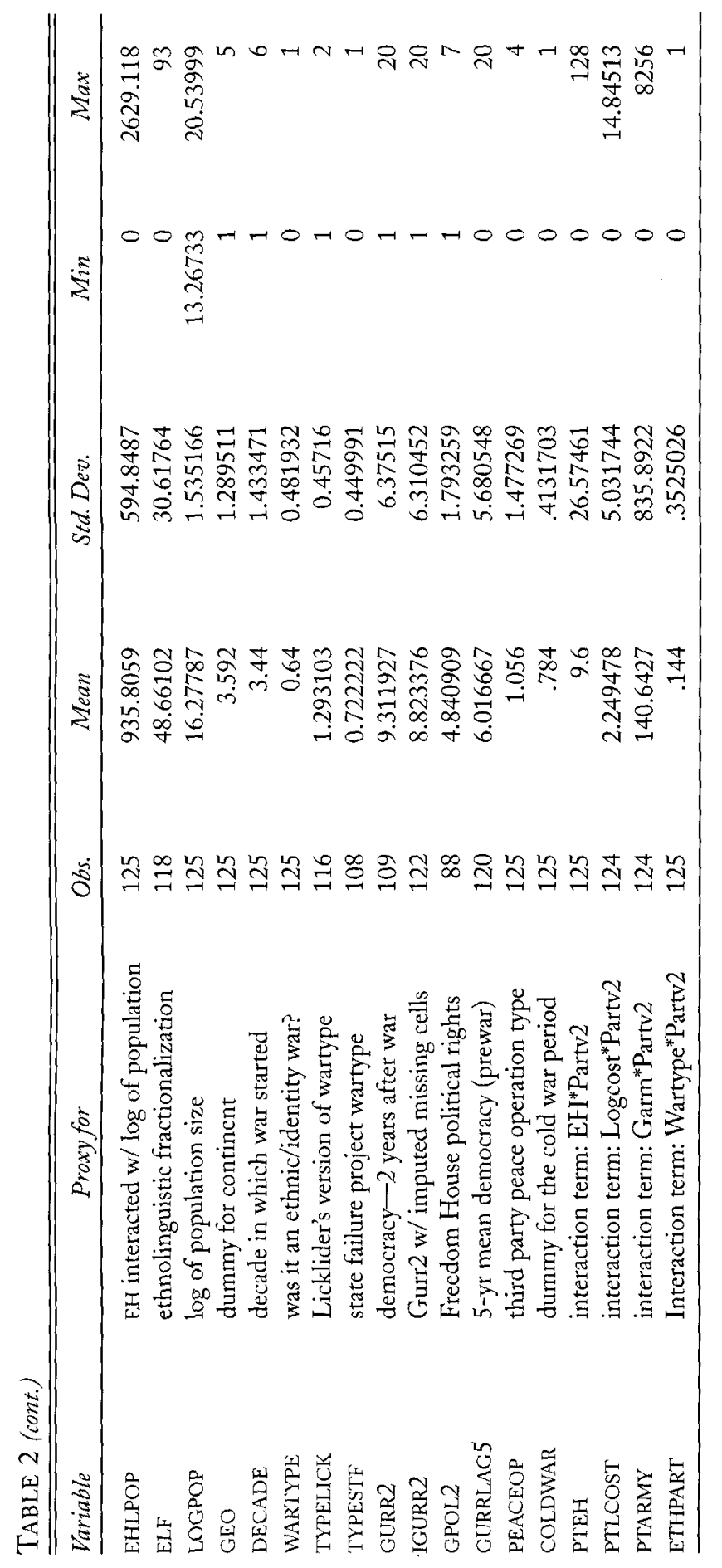




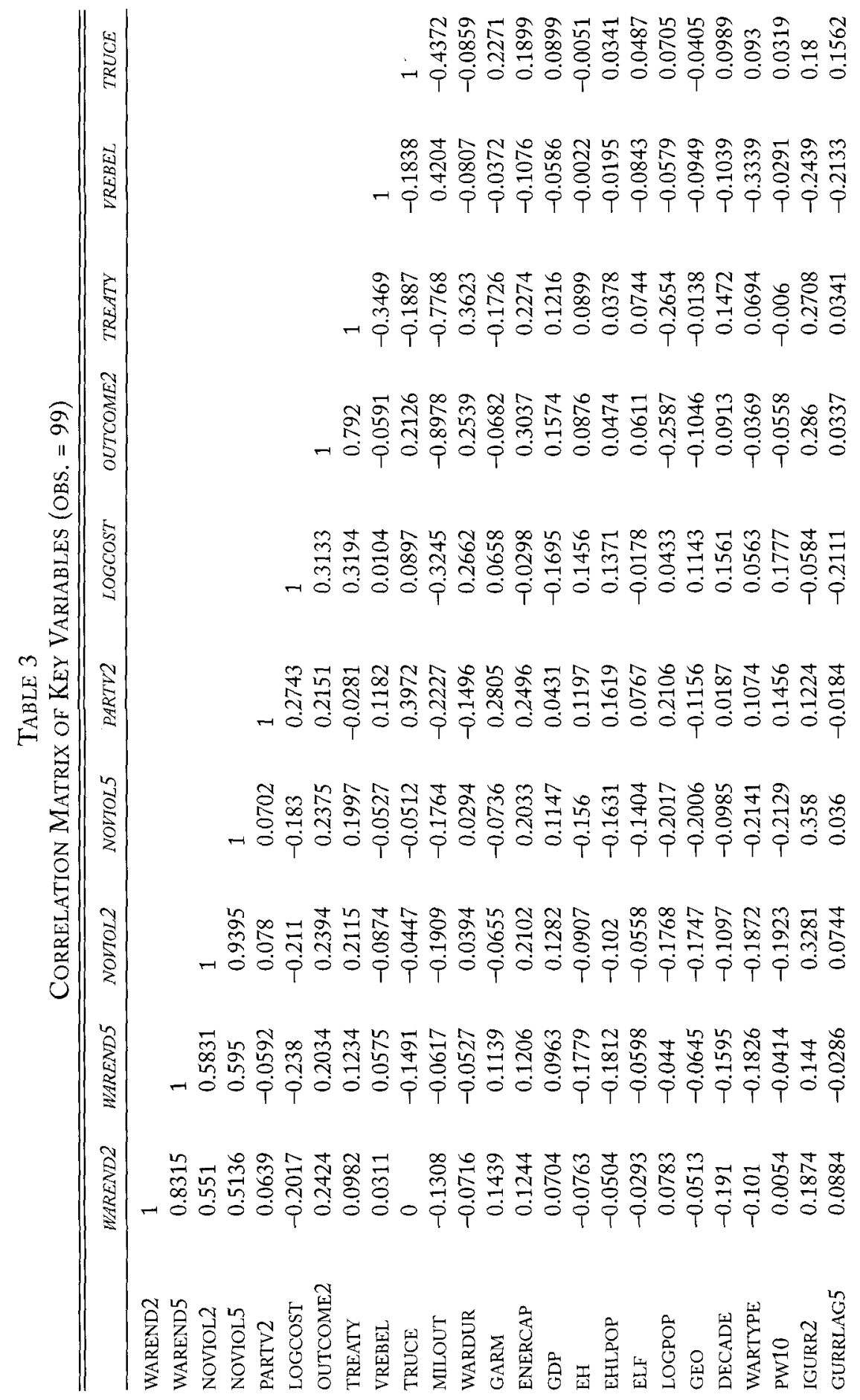




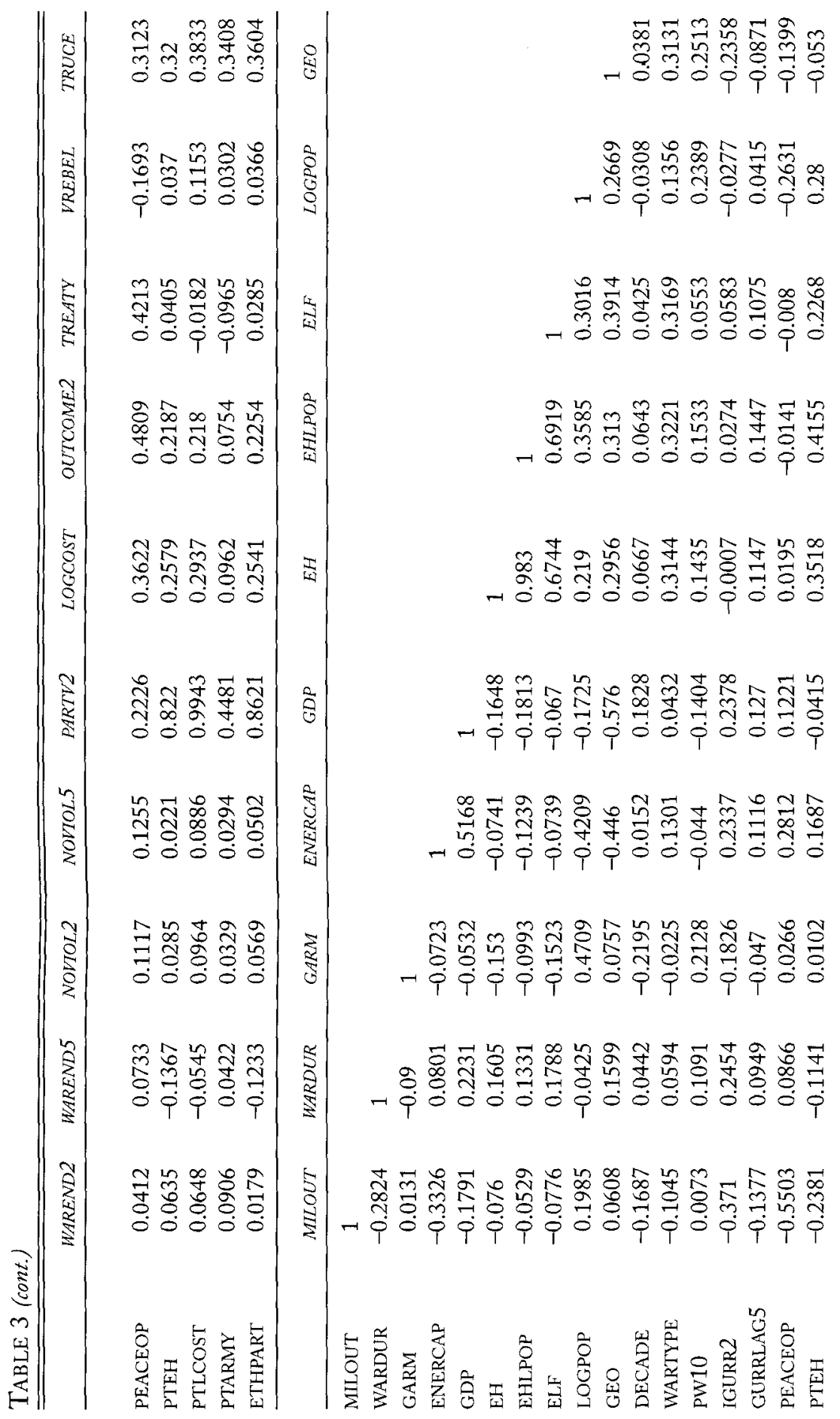




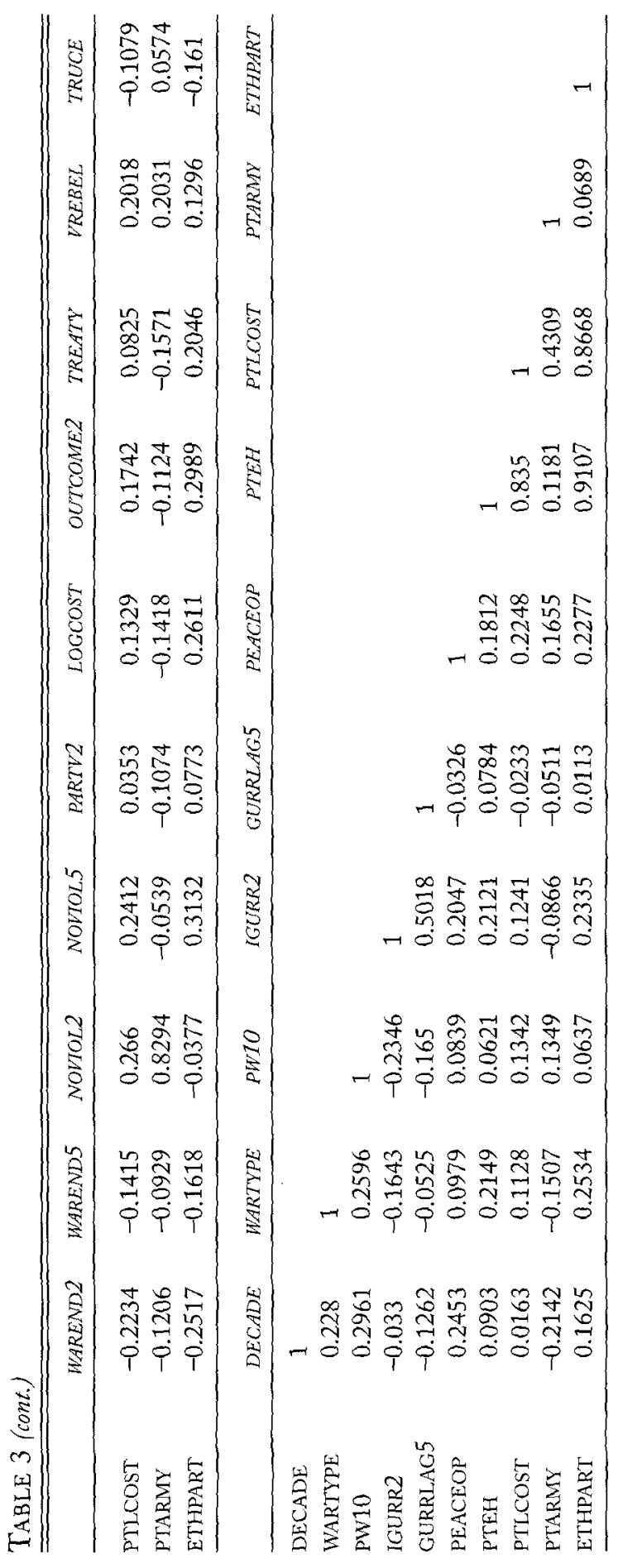


tion. ${ }^{46}$ Partition is not usually the goal of revolutions motivated by ideology, but it is often the stated goal of ethnic war. For the same reason, ethnic heterogeneity (EH) should be significantly associated with partition, especially if ethnic groups are large enough to constitute a politically and economically viable successor state. The human toll of the war (LOGCOST) should be positively correlated with partition. The intuition is that extremely violent wars can be settled only by partition and that the international community might be more supportive of such an outcome in those cases. The outcome of the war (OUTCOME2) should also be a significant determinant of partition, since we would not expect to see partition if the government wins a military victory, whereas partition would be more likely in the case of rebel victory. Population size (LOGPOP) should be positively associated with partition. ${ }^{47} \mathrm{I}$ also include as control variables a number of socioeconomic indicators of the country's overall level of development, since many studies have identified such variables as significant determinants of civil war. ${ }^{48}$ However, it is harder to theorize about the nature of their association with the incidence of partition. I present the results of my estimations in Table 4.

A first important finding is that, as theorized, the type of the war is indeed a significant determinant of partition. Identity wars (ethnic and religious wars) are positively and significantly correlated with partition in model 1. This implies that the partition theorists correctly argue that ethnicity matters for the onset of partition. However, when I tested alternatively coded variables for the type of war in models $1 b$ and $1 c, I$ found a significant difference: both variables were significant, but Licklider's variable (TYPELICK in model 1a) is negatively correlated with the onset of partition whereas the State Failure Project variable (TYPESTF in model $1 \mathrm{~b}$ ) is positively correlated with partition. This implies that, while ethnicity seems important for partition, the direction of their association might be influenced by assumptions made in the coding of the WARTYPE variable. ${ }^{49}$ At the same time, only small changes are ob-

\footnotetext{
${ }^{46}$ The coding of the WARTYPE variable was not easy. There are substantial differences in the various sources and data sets I consulted. I used two main sources for this variable: Licklider (fn. 32); and Esty et al. (fn. 33). I coded the variables TYPELICK (Licklider's war-issue variable) and TYPESTF (the StateFailure Project's war-type variable) to facilitate comparisons across cases. Where those two sources differed, I coded WARTYPE based on majority opinion in other data sets, including Regan (fn. 33); and Mason and Fett (fn. 33).

${ }^{47}$ This hypothesis (with an emphasis on the proportion of young men) has been posited with reference to the causes of civil war by Collier and Hoeffler (fn. 45); and Robert H. Bates, "Ethnicity, Capital Formation, and Conflict," CID Working Paper no. 27 (Harvard University, October 1999).

${ }^{48}$ For example, Collier and Hoeffler (fn. 45).

${ }^{49}$ Given such problems, quantitative analysts of civil wars must be highly transparent in their coding of these variables. Moreover, it is necessary for those building data sets of civil war to coordinate their
} 
TABLE 4

Probit Regression of Occurrence of Partition ${ }^{a}$

\begin{tabular}{|c|c|c|c|c|c|}
\hline Dep. Var: Partition & Model 1 & Model $1 a$ & Model $1 b$ & Model 2 & Model 3 \\
\hline Constant & $\begin{array}{l}-4.53^{* * * *} \\
(1.64)\end{array}$ & $\begin{array}{l}-2.09 \\
(1.82)\end{array}$ & $\begin{array}{r}-3.93^{*} \\
(2.12)\end{array}$ & $\begin{array}{l}-6.72^{*} \\
(3.48)\end{array}$ & $\begin{array}{l}-2.38 \\
(2.09)\end{array}$ \\
\hline $\begin{array}{l}\text { WARTYPE } \\
\text { (identity or } \\
\text { ideology?) }\end{array}$ & $\begin{array}{l}1.65^{* * *} \\
(.553) \\
.103\end{array}$ & - & - & $\begin{array}{l}2.22^{* * *} \\
(.822) \\
2.22\end{array}$ & $\begin{array}{l}1.59^{* * *} \\
(.547) \\
.032\end{array}$ \\
\hline $\begin{array}{l}\text { LOGCOST } \\
\text { (log of deaths \& } \\
\text { displacements }\end{array}$ & $\begin{array}{l}.358^{* * * *} \\
(.118) \\
.025\end{array}$ & $\begin{array}{l}.471^{* * *} \\
(.141) \\
.025\end{array}$ & $\begin{array}{l}.338^{* *} \\
(.138) \\
.034\end{array}$ & $\begin{array}{l}.441^{* * *} \\
(.152) \\
.44\end{array}$ & $\begin{array}{l}.396^{* * *} \\
(.138) \\
.008\end{array}$ \\
\hline $\begin{array}{l}\text { EH } \\
\text { (Index of Ethnic } \\
\text { Heterogeneity) }\end{array}$ & $\begin{array}{l}-.064^{* *} \\
(.026) \\
-.005\end{array}$ & $\begin{array}{l}-.068^{* *} \\
(.029) \\
-.004\end{array}$ & $\begin{array}{l}-.063^{* *} \\
(.027) \\
-.006\end{array}$ & $\begin{array}{l}-.049 \\
(.035) \\
-.049\end{array}$ & $\begin{array}{l}-.075^{* * *} \\
(.027) \\
-.0015\end{array}$ \\
\hline $\begin{array}{l}\text { EHLPOP } \\
\quad\left(\mathrm{EH}^{*} \log \text { of }\right. \\
\text { population size })\end{array}$ & $\begin{array}{l}.003^{* *} \\
(.001) \\
.0002\end{array}$ & $\begin{array}{l}.0037^{* *} \\
(.002) \\
.0002\end{array}$ & $\begin{array}{l}.0034^{* *} \\
(.0015) \\
.0003\end{array}$ & $\begin{array}{c}.003 \\
(.002) \\
.003\end{array}$ & $\begin{array}{r}.004^{* * *} \\
(.0014) \\
.00008\end{array}$ \\
\hline $\begin{array}{l}\text { TRUCE } \\
\text { (war ended in an } \\
\text { informal truce?) }\end{array}$ & $\begin{array}{l}1.69^{* * *} \\
(.348) \\
.337\end{array}$ & $\begin{array}{l}1.76^{* * *} \\
(.383) \\
.313\end{array}$ & $\begin{array}{c}1.71^{* * *} \\
(.342) \\
.401\end{array}$ & $\begin{array}{l}1.58^{* * *} \\
(.438) \\
1.58\end{array}$ & $\begin{array}{l}1.75^{* * *} \\
(.414) \\
.183\end{array}$ \\
\hline $\begin{array}{l}\text { VREBEL } \\
\text { (war ended with a } \\
\text { rebel victory?) }\end{array}$ & $\begin{array}{c}1.55^{* * *} \\
(.519) \\
.247\end{array}$ & $\begin{array}{l}1.59^{* * *} \\
(.578) \\
.214\end{array}$ & $\begin{array}{c}1.35^{* * *} \\
(.425) \\
.256\end{array}$ & $\begin{array}{l}1.91^{* *} \\
(.778) \\
1.91\end{array}$ & $\begin{array}{l}2.16^{* * *} \\
(.502) \\
.229\end{array}$ \\
\hline $\begin{array}{l}\text { GEO } \\
\text { (continent) }\end{array}$ & $\begin{array}{l}-.594^{* * * *} \\
(.172) \\
-.042\end{array}$ & $\begin{array}{l}-.644^{* * *} \\
(.205) \\
-.035\end{array}$ & $\begin{array}{l}-.651^{* * *} \\
(.192) \\
-.065\end{array}$ & $\begin{array}{l}-.514^{* *} \\
(.207) \\
-.513\end{array}$ & $\begin{array}{l}-.405^{*} \\
(.225) \\
-.008\end{array}$ \\
\hline $\begin{array}{l}\text { DECADE } \\
\text { (decade in which } \\
\text { war started) }\end{array}$ & $\begin{array}{l}-.175 \\
(.140) \\
-.012\end{array}$ & $\begin{array}{l}-.204 \\
(.161) \\
-.011\end{array}$ & $\begin{array}{l}-.167 \\
(.151) \\
-.017\end{array}$ & $\begin{array}{l}-.019 \\
(.181) \\
-.019\end{array}$ & $\begin{array}{l}-.023 \\
(.153) \\
-.0005\end{array}$ \\
\hline $\begin{array}{l}\text { TYPELICK } \\
\text { (Licklider's wartype) }\end{array}$ & & $\begin{array}{c}-2.07^{* * *} \\
(.591) \\
-.113\end{array}$ & & & \\
\hline $\begin{array}{l}\text { TYPESTF (State } \\
\text { Failure } \\
\text { Project wartype) }\end{array}$ & & & $\begin{array}{c}1.32^{* *} \\
(.576) \\
.094\end{array}$ & & \\
\hline $\begin{array}{l}\text { ILLIT (percentage } \\
\text { population } \\
\text { that is illiterate) }\end{array}$ & & & & $\begin{array}{l}-.021 \\
(.016) \\
-.02\end{array}$ & \\
\hline $\begin{array}{l}\text { GDP (real GDP } \\
\text { per capita, PPP) }\end{array}$ & & & & $\begin{array}{l}2.17 \mathrm{e}-07 \\
(.0001) \\
2.17 \mathrm{e}-07\end{array}$ & \\
\hline $\begin{array}{l}\text { LIFES (life } \\
\text { expectancy at birth) }\end{array}$ & & & & $\begin{array}{l}-.002 \\
(.039) \\
-.002\end{array}$ & \\
\hline $\begin{array}{l}\text { GINI (income } \\
\text { inequality index) }\end{array}$ & & & & & $\begin{array}{l}-.113^{* * *} \\
(.029) \\
-.002\end{array}$ \\
\hline $\begin{array}{l}\text { ENERGYS (energy } \\
\text { consumption } \\
\text { per capita) }\end{array}$ & & & & & $\begin{array}{c}.0003 \\
(.0002) \\
5.72 \mathrm{e}-06\end{array}$ \\
\hline
\end{tabular}


TABLE 4 (cont.)

\begin{tabular}{lccccc}
\hline Dep. Var. Partition & Model 1 & Model 1a & Model 1b & Model 2 & Model 3 \\
\hline Observations & 124 & 115 & 107 & 116 & 123 \\
Log-likelihood & -30.365 & -27.397 & -29.242 & -22.042 & -21.529 \\
Pseudo-R & 0.4616 & 0.4844 & 0.4481 & 0.5597 & 0.6170 \\
Correctly classified & $87.20 \%$ & $87.82 \%$ & $86.91 \%$ & $92.24 \%$ & $93.49 \%$ \\
Reduction in error & $23.80 \%$ & $27.55 \%$ & $22.08 \%$ & $53.80 \%$ & $61.25 \%$ \\
\hline
\end{tabular}

*** significant at the .01 level; ** significant at the .05 level; * significant at the .10 level (two-tailed tests)

${ }^{a}$ Reported are coefficients (robust standards errors) and marginals ( $\mathrm{dF} / \mathrm{dx}$ ), in that order.

served in most of the other variables in models $1 \mathrm{a}$ and $1 \mathrm{~b}$, which suggests that the other variables are robust.

Ethnicity has a complicated relationship with partition. In models 1 , $1 \mathrm{a}$, and $1 \mathrm{~b}, \mathrm{I}$ found that as ethnic heterogeneity increases, the probability of a partition decreases significantly, suggesting that it may be difficult to coordinate and win in a secessionist war in extremely diverse societies. ${ }^{50}$ However, as the size of ethnic groups increases, so does the probability of partition. ${ }^{51}$ Large ethnic groups may be better able to overcome the coordination problems associated with mounting a rebellion and better able to defend their territory.

As expected, I found that partitions are positively and significantly correlated with the level of violence (LOGCOST). This variable is very robust, and since deaths and displacements chronologically precede the occurrence of partition, these results may be pointing to a causal relationship between high levels of violence and partition. At the same time, my data do not allow me to preclude the possibility that some of the observed violence may actually have been caused by partition (as, for example, in India and Cyprus).

War outcomes are also significant in model 1 and all its variants. Both rebel victory and truce are significantly and positively associated with the incidence of partition (although, these results should be interpreted with caution due to high collinearity among these regressors).

In model 2 I added several socioeconomic variables that may have been important causes of the previous war and I found them individually insignificant. While I did not expect to find individual significance

efforts and exchange information. I will use my WARTYPE variable in the rest of the analysis since I generated it with reference to as many sources as I could consult for each case. I tried to reflect majority opinion about the coding of each case, where there was disagreement between my main sources.

${ }^{50}$ If we drop WARTYPE, then EH becomes nonsignificant but remains negative.

${ }^{51}$ I proxy the size of ethnic groups by interacting the ethnic heterogeneity index $\mathrm{EH}$ with the $\log$ of population size (LOGPOP). 
given their high level of collinearity, I did expect and did find joint significance (a joint test of ILLIT, LIFES, GDP, DECADE, and GEO yielded $\operatorname{chi} 2(5)=11.85$ and Prob $>\operatorname{chi} 2=0.0369$ ). The fact that these variables are not individually significant allows me to use them as controls in my analytical models of war recurrence in the next section, since they are not significantly correlated with partition and I use partition as a core regressor in those models. The most important impact of these local capacity variables is that they make ethnicity (EH, EHLPOP) nonsignificant (although this may be due to the noise that they introduce in the equation).

Finally, in model 3 I controlled for income inequality (GINI) and replaced the socioeconomic controls of model 2 with a variable measuring the country's overall level of economic development (ENERGYS) at the start of the war. ${ }^{52}$ Here I found a positive but nonsignificant relationship between economic development and partition. The opposite association is often found between development and the risk of onset of war, but my finding makes sense, since low levels of economic development often discourage ethnic minorities from seceding (although a more accurate result might have been obtained if I had data available on the regional concentration of natural resources and the geographic dispersion of industries within each country). ${ }^{53}$ Income inequality is significant but negatively correlated with partition, which once again seems counterintuitive. ${ }^{54}$ The control variables for geographical location and for the decade during which the war started pick up time- and place-specific effects. The DECADE variable is nonsignificant, but there seem to be important regional effects (which could be better studied in the context of a panel data set).

To summarize, I found that partition is significantly more likely to occur after an identity war than after an ideological war, after an informal truce or rebel victory following a very costly war, in a country with

\footnotetext{
${ }_{52}$ Measuring these variables at the start of the war not only prevents problems of reverse causality but also captures any impact that these variables might have had on causing the civil war in the first place. Thus, the inability to find significance for the economic variables in model 2 may be due to a selection effect (since all the countries in my sample are countries that experienced war and may therefore share the same socioeconomic background). Thus, the analysis of partition and war recurrence must focus here on war-related variables that would be expected to differ significantly across countries.

${ }^{53}$ See Collier and Hoeffler (fn. 45). The precise relationship between partition and economic variables is undertheorized, so I will not explore this further, but this counterintuitive finding is worth further study. It may be that relatively richer countries can support partition, since the prospects of economic viability of the successor state will be greater.

${ }^{54}$ These signs do not change if I drop the cases of ongoing war. The direction of this correlation, however, may result from measurement error or selection effects. Measurement error is possible because reliable data were often not available for the relevant years. Or it may be due to collinearity between income inequality and energy consumption, since I used GDP data to impute missing values of both of these variables.
} 
large ethnic groups and small levels of ethnic heterogeneity and a relatively higher level of economic development (compared to other wartorn states). Having identified these correlates of partition, I can now test the three critical hypotheses of partition theory: (1) that partitions create successor states that are at least as democratic as their predecessors, if not more so; (2) that partitions reduce the risk of war recurrence; and (3) that partitions reduce low-level ethnic violence after the war ends.

\section{Testing The Hypotheses.}

\section{Does Partition Create Undemocratic States?}

Kaufmann has argued that successor states are generally no less democratic than their predecessors and that they can even be more democratic. ${ }^{55}$ Although a full test of this hypothesis is not yet possible (see below), a first cut at such a test is provided in this section.

Using data on democracy and autocracy from the Polity 98 data set, I have created the variable GURR, measuring the level of democracy two years (GURR 2) and five years (GURR 5) after the end of the war for both predecessor and successor states. ${ }^{56}$ Partitioned countries have a mean and standard deviation of GURR 2 of 11.02 and 6.204, respectively. The corresponding values for nonpartitioned countries are 8.42 and 6.27 , respectively. A score of 20 suggests a perfect democracy, whereas a score of 0 , an extreme autocracy. The computed averages reveal that countries that have experienced a civil war-regardless of whether or not they have been partitioned-are generally nondemocratic two years after the end of the war. This may be due to the war itself or to a legacy of undemocratic institutions. These legacies can be measured by GURRLAG5-the mean level of democracy during the five years prior to the start of the war. Table 5 lists all partitioned countries and their GURR and GURRLAG5 indices. Thirteen cases broadly support Kaufmann's hypothesis that partitioned countries are no less democratic than their predecessors and six cases do not support that hypothesis. ${ }^{57}$

${ }^{55}$ Kaufmann (fn. 1, 1998), 124.

${ }^{56}$ The original data on democracy were compiled by Keith Jaggers and Ted Robert Gurr, polity 98 Project (http://www bsos.umdedu/cidcm/polity/). I added their democracy and autocracy scores as follows: $\mathrm{GURR}=[\mathrm{DEMOCRACY}+(10-A U T O C R A C Y)]$. The resulting variable ranges from 0 to 20 . The Polity3 data end in 1994, so I imputed thirty-five missing values using the political rights index of the Freedom House project after I established that there was a very close correlation between Gurr's democracy index and Freedom House's political rights index. See Freedom House, Freedom in the World (London: Freedom House, 1999).

57 This list includes not only ethnic partitions but also all other cases of partition in my data set. Subsequent analysis focuses directly on partitions that resulted from ethnic wars and therefore excludes a number of partitions (for example, the Koreas, Vietnam, and Taiwan). However, I test the robustness of my results by including all wars and partitions. 
TABLE 5

PRewar ANd PostPartition Polity INDices ${ }^{2}$

\begin{tabular}{lcc}
\hline \hline Country Name & $\begin{array}{c}\text { Five-Year Prewar } \\
\text { Democracy Index } \\
\text { (GURRLAG5) }\end{array}$ & $\begin{array}{c}\text { Democracy Index } \\
\text { Two Years after the War } \\
\text { (GURR2) }\end{array}$ \\
\hline Azerbaijan & 3 & 4 \\
Yugoslavia-Bosnia & 6 & 9 \\
China-Taiwan & 5 & 2 \\
Yugoslavia-Croatia & 6 & 11 \\
Yugoslavia-Croatia & 6 & 9 \\
Cyprus & 1 & - \\
Cyprus & 1 & 20 \\
Ethiopia-Eritrea & 1 & 11 \\
Georgia-Abkhazia & 11 & 15 \\
Georgia-Ossetia & 11 & 15 \\
India-Pakistan & 1 & 16 \\
India-Kashmir & 19 & 17 \\
India-Kashmir & 18 & 18 \\
Israel-Palestine & - & - \\
Korea (North-South) & 1 & 3 \\
Moldova & 1 & 17 \\
Pakistan-Bangladesh & 3 & 18 \\
Russia-Chechnya & 11 & 14 \\
Somalia-Somaliland & 3 & $2^{*}$ \\
Tajikistan & 11 & 5 \\
Vietnam, Republic of & 7 & 3 \\
\hline
\end{tabular}

${ }^{a}$ GURR2 includes one imputed value (Somalia), denoted with an asterisk and rounded to the nearest integer. If the predecessor state was a colony or not a recognized state (e.g., Israel) 1-5 years before the war started, then we do not have a Polity score since these are only available for independent states. In those cases, I have entered an N/A and comparison of the polity index before and after the war is not possible. The same is true for cases where the country was created out of the civil war, as in Israel. A dot indicates no available information. The index is based on data from Polity 98 (see text).

An equality of means test for both GURRLAG5 and GURR2 reveals signs of significant differences between partitioned and nonpartitioned countries. Specifically, a t-test of the null hypothesis that there is no significant difference in the mean of GURR2 in partitioned and nonpartitioned countries can marginally be rejected with 120 degrees of freedom and a $t$-statistic of $-1.6644(\mathrm{P}>|\mathrm{t}|=0.098)$. A two-sample $\mathrm{t}$-test with equal variances only on cases of ethnic war does reveal that there is a significant difference and that partitioned countries have higher democracy averages (75 d.f.; $t=-3.3842$ ). However, this effect need not refer to the democratization effect of partition and may be due to the prewar level of democracy, which is on average higher among 
partitioned countries that have experienced war. A means test with respect to $\mathrm{DEMCH}$ - a variable that measures the difference of postwar and prewar levels of democracy-rejects equality with 73 degrees of freedom and $t=-2.6597(P>|t|=0.0096)$. These results suggest that partitioned states fare slightly better in terms of postwar democratization.

I turn next to a multivariable OLS regression of GURR2 (and GURR5) using partition as one of the explanatory variables. A bivariate regression of democracy on partition shows no significance of partition at the 5 percent level if we use the entire data set but finds partition positive and significant among cases of ethnic war. A clearer picture emerges from multivariate models (see Table 6). In model $1 \mathrm{I}$ regressed the postwar democracy index (with imputed missing values) on a number of explanatory variables, including partition. ${ }^{58}$ Most of the variance in the dependent variable in this and the other models is explained by the country's democratic legacy (GURRLAG5), which is extremely robust and significantly increases postwar levels of democracy. There is a weak positive correlation between democratic postconflict states and the presence of third-party peace operations (PEACEOP). Given that this association is weak, I dropped PEACEOP from subsequent regressions. There is a negative, though not very robust relationship between the level of democracy and the size of the government's military, which suggests that troop demobilization and force reductions in postconflict states may be useful in promoting peace and democracy. War duration is significant and positively correlated with the level of democracy, lending support to the war-weariness hypothesis (that people tired of war will try harder to build peace). Real per capita GDP is positively correlated with democracy (as would be expected), but this association is not significant, possibly due to selection effects or measurement error. ${ }^{59}$ Partition is positively correlated with democracy and it is significant at the 10 percent level. The type of war is also significant and the coefficient sign suggests that ethnic wars would reduce the postwar mean of democracy by 2.5 points as compared with nonethnic wars.

In model 2 I looked more closely at cases of ethnic war, and the results discussed previously are nearly identical, except for the signifi-

${ }^{58}$ I selected these variables based on theoretical arguments regarding the determinants of the level of democratization after civil war, drawing on Doyle and Sambanis (fn. 45), among others. Also the relationship between economic variables and democracy has been the focus of numerous studies in the economics and political science literatures; see, e.g., Ross E. Burkhart and Michael S. Lewis-Beck, "Comparative Democracy: The Economic Development Thesis," American Political Science Review 88 (December 1994).

${ }^{59}$ These regression results are robust for a large number of specifications. 


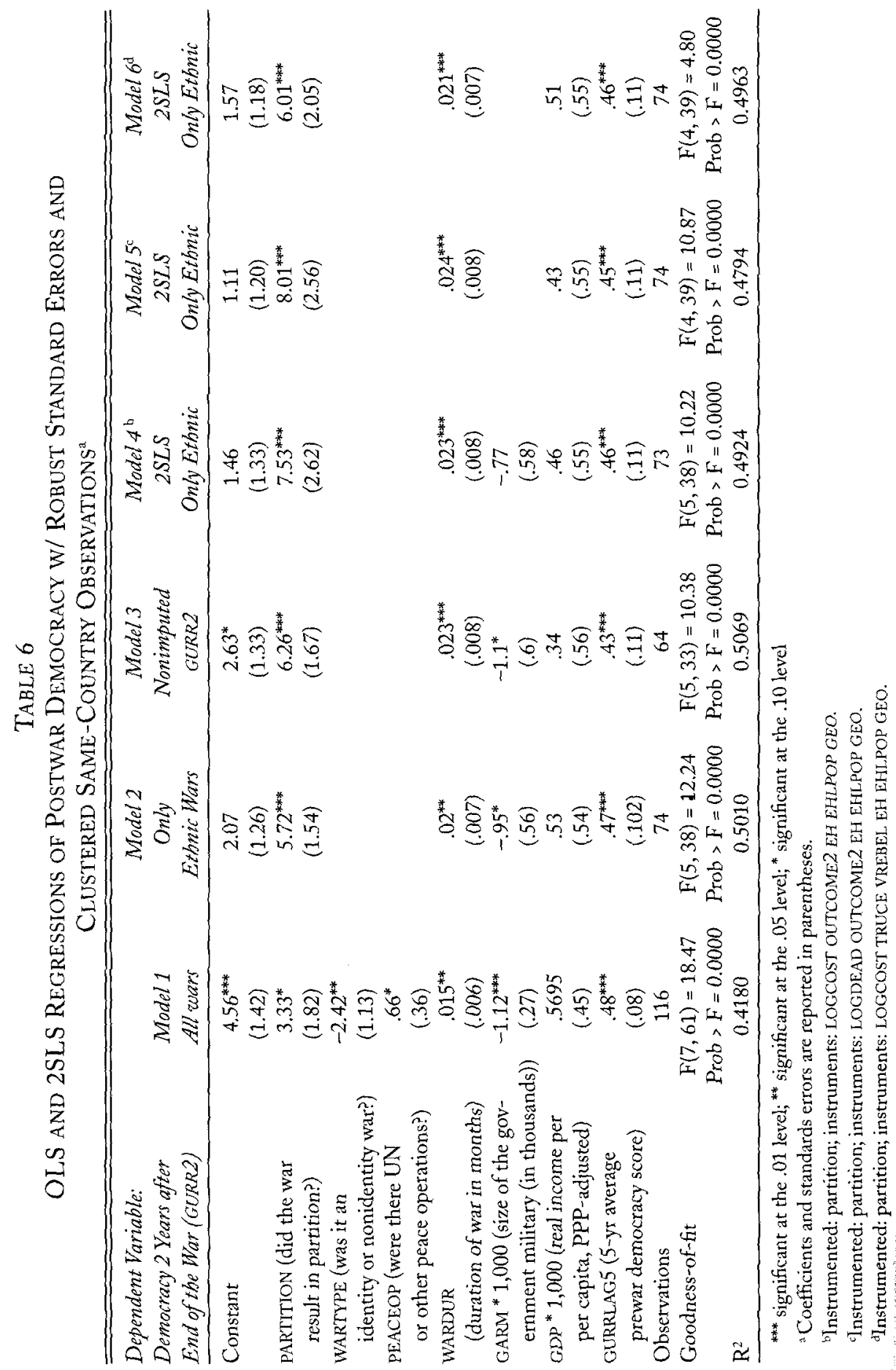


cance and coefficient of partition, which now more than doubles. In model 3 I regressed the same right-hand-side variables on the GURR2 variable without imputing missing values, and the results are robust. The democratic legacy variable (GURRLAG5) seems to be doing heavy lifting in these regressions. If we drop it from models $2-3$, the $\mathrm{R}^{2}$ drops by about 20 percentage points and the coefficient of partition increases by 20 percent.

Partition may also be endogenous or, rather, jointly determined with some of the other right-hand-side variables. Thus, I reestimated model 2 using a two-stage least squares (2SLS) estimator; no significant differences were observed (both the coefficient and standard error of partition were extremely volatile, but the t-test remained the same). In model 5 I estimated a different specification of this model again using 2 SLS, dropping the now nonsignificant GARM variables, but again no major change occurred. In model 6 I changed two of the instrumental variables used in the first-stage regressions. Throughout these changes in specification and estimation method, the democratic legacy variable (GURRLAG5) was extremely robust, as was partition. The coefficient of the partition variable was positive and varied between 6 and 8 .

Finally, testing the hypothesis that the instrumented 2SLS models might be more efficient than the OLS models, I found the OLS models more efficient. A Hausman test of the null hypothesis that the difference in coefficients between the instrumented and OLS models is not systematic, estimated as: $(b-B)^{\prime}\left[\left(V_{b}-V_{B}\right)^{-1}\right](b-B)$, yielded a chisquare statistic of 0.57 with 4 degrees of freedom, so we cannot reject the null hypothesis that the instrumented models are less efficient and we should rely on the OLS estimates of models $1-3 .^{60}$

Overall, these regression results seem to support partition theory. However, this is at best an incomplete picture, since we do not have comparable democracy data for successor states that are not internationally recognized. This therefore creates a problem of systematic bias in the coding of the dependent variable, which reduces the reliability of the findings just described. Successor states that are not internationally recognized may well have low democracy; contrast, for example, the political institutions of the Republic of Cyprus with those of the socalled Turkish Republic of Northern Cyprus or the democracy levels in Chechnya versus those in Russia or in Ossetia and Abkhazia versus

${ }^{60} \mathrm{~J}$. Hausman, "Specification Tests in Econometrics," Econometrica 46 (1978), 1251-71. On the use of the Hausman test to test exogeneity, see B. H. Baltagi, Econometrics (New York: Springer-Verlag, 1998), 291; and Stata Corporation, Stata Reference Manual: Release 6 (College Station, Tex.: Stata, 1999), 2:7-13. In the formula above $b$ is the coefficient vector from the consistent estimator and $B$ the coefficient vector from the efficient estimator and $V_{b}$, and $V_{B}$ are their respective covariance matrices. 
those in Georgia. These comparisons are difficult, given that the Polity 98 project does not provide information on these territories. Thus, before making a judgment on the democratizing effects of partition, we need comparable data on democracy for all successor and predecessor states ${ }^{61}$ At this stage, I can neither reject nor accept the claims of the partition theorists with confidence. The link between partition and democratization must be studied further as high-quality data become available. At that time it would also be interesting to see whether high levels of GURRLAG5 tend to promote democracy in postwar successor states. At this stage the robustness of GURRLAG5 suggests that our focus should not be on designing democracy-friendly partitions but rather should be on strengthening democratic institutions in countries before they actually experience a civil war.

I now turn to the critical question of war recurrence.

\section{Does Partition Prevent War Recurrence?}

Several examples of wars following partitions provide support for the critics of partition theory: Croatia fought a second war with Serbia after it was partitioned in 1991. Ethiopia and Eritrea fought a bitter territorial war in 1999-2000 after being partitioned in 1991. The partition of Somaliland collapsed in a wave of new violence in 1992. India and Pakistan have fought three wars since their partition in 1947. Cyprus was at war again in 1974 after it was effectively partitioned into militarily defensible, self-administered enclaves between 1963 and 1967. ${ }^{62}$ At the same time, seemingly intractable conflicts and bloody ethnic wars have given way to peace without partition, as, for example, in South Africa, Guatemala, and Uganda.

${ }^{61}$ Given the paucity of data to answer this important question, a worthwhile project would be to conduct a comparative case study of the political institutions of all these successor states.

${ }^{62}$ This is not a well-known case. In 1963 a "green line" was established in the capital city of Nicosia, partitioning the Greek Cypriot and Turkish Cypriot sectors. After 1964 the partition was expanded, and more than 30 percent of the Turkish Cypriot population moved to defensible, self-administered enclaves. These enclaves forcibly excluded the Greek Cypriot population and their demilitarization and defortification was part of the mandate given to a UN peacekeeping force-UNFICYP (see UN doc. S/5764, 15 June 1964, para. 61). The UN secretary-general often noted in his report that the enclaves gave the Turkish Cypriots "complete military and administrative control" of several areas (S/6228, para. 50). In 1965 the secretary-general noted that the enclave fortifications "contribute to maintaining tension at high pitch" and UNFICYP "insists on their removal" (S/6228, para. 51). Within six months in 1967, 52 new positions were built by the Greek Cypriot National Guard and 130 by the Turkish Cypriots (S/8286, December 8, 1967, para. 50). The secretary-general noted that "this ceaseless building of fortifications ... [would] result in the Island being criss-crossed and honeycombed with defences [sic]" (S/8286, para. 49). Thus, the island was effectively partitioned between 1963 and 1967. On the Cyprus conflict during the critical years between 1963 and 1974, see Richard Patrick, Political Geography and the Cyprus Confict, 1963-1971 (Waterloo, Canada: Department of Geography, University of Waterloo, 1976); the work includes maps of the pre-1974 enclaves. See also Joseph Joseph, Cyprus: Ethnic Conflict and International Solution (New York: St. Martin's, 1997); and Sambanis (fn. 33). 
TABLE 7

Cross-Tabs between Partition, War Recurrence, and

RESIDUAL VIOLENCE

(TWO, FIVE, AND TEN YEARS AFTER THE WAR)

\begin{tabular}{|c|c|c|c|c|c|}
\hline $\begin{array}{l}\text { War } \\
\text { Ended for } \\
\end{array}$ & $\begin{array}{c}\text { War } \\
\text { Recurrence }\end{array}$ & Partitition & $\begin{array}{c}\text { No } \\
\text { Partitition }\end{array}$ & $\begin{array}{l}\text { Total } \\
\text { Cases }\end{array}$ & $\begin{array}{c}\text { Pearson } \\
\text { Cbi-Square Test } \\
\text { (1 Degree of Freedom) }\end{array}$ \\
\hline 2 years & $\begin{array}{l}\text { war recurred } \\
\text { no war }\end{array}$ & $\begin{array}{l}5 \\
16\end{array}$ & $\begin{array}{l}27 \\
77\end{array}$ & $\begin{array}{l}32 \\
93\end{array}$ & $\begin{array}{l}\text { Pearson chi2 }(1)= \\
0.0425 ; \operatorname{Pr}=0.837\end{array}$ \\
\hline 5 years & $\begin{array}{l}\text { war recurred } \\
\text { no war }\end{array}$ & $\begin{array}{c}5 \\
14\end{array}$ & $\begin{array}{l}24 \\
71\end{array}$ & $\begin{array}{l}29 \\
85\end{array}$ & $\begin{array}{l}\text { Pearson } \operatorname{chi} 2(1)= \\
0.0092 ; \operatorname{Pr}=0.923\end{array}$ \\
\hline 10 years & $\begin{array}{l}\text { war recurred } \\
\text { no war }\end{array}$ & $\begin{array}{l}5 \\
6\end{array}$ & $\begin{array}{l}24 \\
46\end{array}$ & $\begin{array}{l}29 \\
52\end{array}$ & $\begin{array}{l}\text { Pearson } \operatorname{chi} 2(1)= \\
0.5159 ; \operatorname{Pr}=0.473\end{array}$ \\
\hline $\begin{array}{l}\text { War } \\
\text { Ended for }\end{array}$ & $\begin{array}{l}\text { Residual } \\
\text { Violence }\end{array}$ & Partitition & $\begin{array}{c}\text { No } \\
\text { Partitition }\end{array}$ & $\begin{array}{l}\text { Total } \\
\text { Cases }\end{array}$ & $\begin{array}{c}\text { Pearson } \\
\text { Chi-Square Test } \\
\text { (1 Degree of Freedom) }\end{array}$ \\
\hline 2 years & $\begin{array}{l}\text { low violence } \\
\text { no violence }\end{array}$ & $\begin{array}{l}10 \\
11\end{array}$ & $\begin{array}{l}56 \\
48\end{array}$ & $\begin{array}{l}66 \\
59\end{array}$ & $\begin{array}{l}\text { Pearson } \operatorname{chi} 2(1)= \\
0.2719 ; \operatorname{Pr}=0.602\end{array}$ \\
\hline 5 years & $\begin{array}{l}\text { low violence } \\
\text { no violence }\end{array}$ & $\begin{array}{c}9 \\
10\end{array}$ & $\begin{array}{l}48 \\
47\end{array}$ & $\begin{array}{l}57 \\
57\end{array}$ & $\begin{array}{l}\text { Pearson chi2 } 2(1)= \\
0.0632 ; \operatorname{Pr}=0.802\end{array}$ \\
\hline 10 years & $\begin{array}{l}\text { low violence } \\
\text { no violence }\end{array}$ & $\begin{array}{l}8 \\
5\end{array}$ & $\begin{array}{l}38 \\
32\end{array}$ & $\begin{array}{l}46 \\
37\end{array}$ & $\begin{array}{l}\text { Pearson chi2 }(1)= \\
0.2334 ; \operatorname{Pr}=0.629\end{array}$ \\
\hline
\end{tabular}

These examples, however, do not constitute sufficient proof that the critics are right. To develop better insight into the relationship between partition and war recurrence, I present simple cross-tabulations of partition and war recurrence and low-level violence in Table $7 .{ }^{63}$ No statistical association between partition and ending violence is evident either for the entire population of cases or for ethnic wars.

A fuller test of the relationship between partition and war recurrence, conditional on the effects of several other variables, can be derived by estimating multivariable regressions (see Table 8). The dependent variable is WAREND (did the war end?) with the suffixes 2, 5, and 10 denoting that it is observed two, five, and ten years after the end of the civil war. WAREND is coded 1 if there is no war recurrence and 0 otherwise. ${ }^{64}$ The control variables in these regressions were selected on

${ }^{63}$ This table includes cases of nonethnic partition. I also ran these cross-tabs excluding nonidentity (ethnic/religious) wars, and the results were not significantly affected.

${ }^{64}$ I estimate probit models with clustered same-country observations and robust standard errors. Since partition theory has focused on ethnic wars and since I found the type of war to be a significant determinant of partition, I dropped cases of nonidentity wars from my analysis, but I do report some results of interest as they apply to all civil wars. 


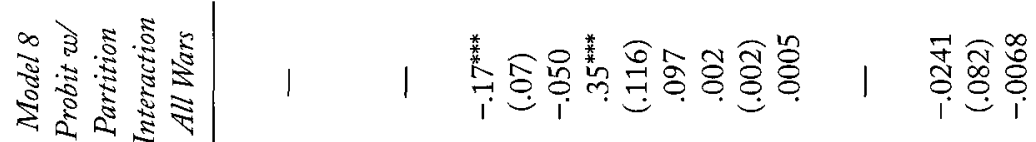

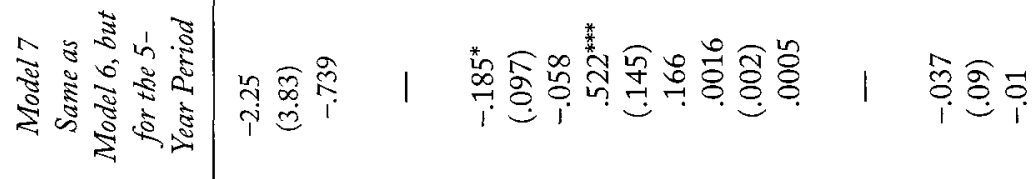

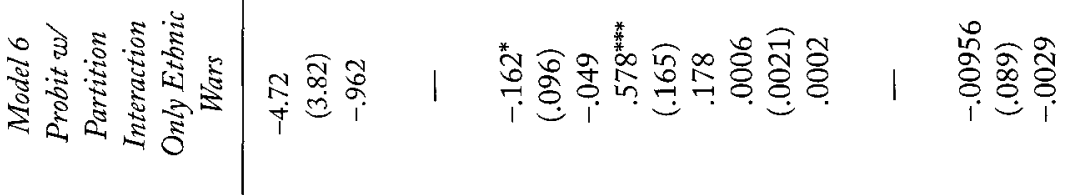

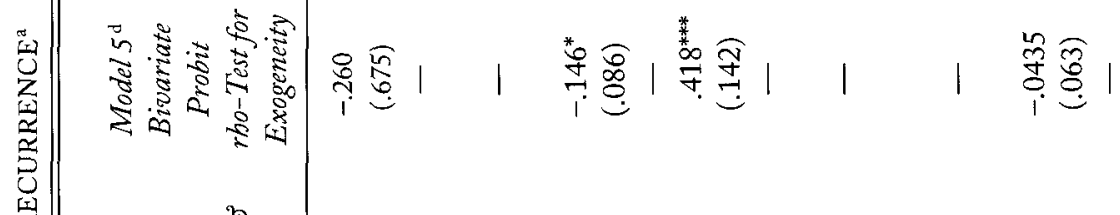

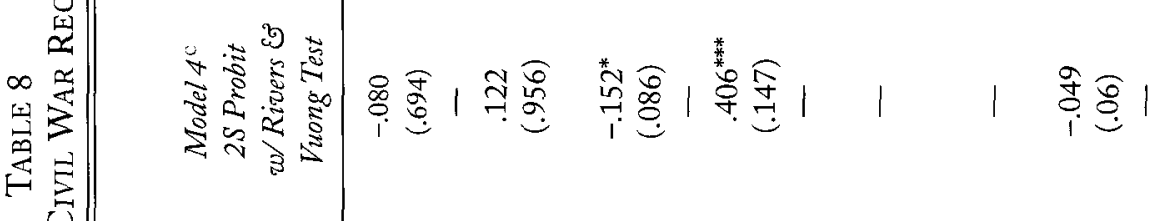

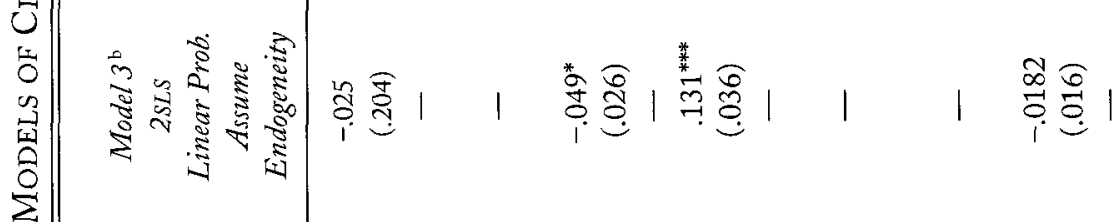

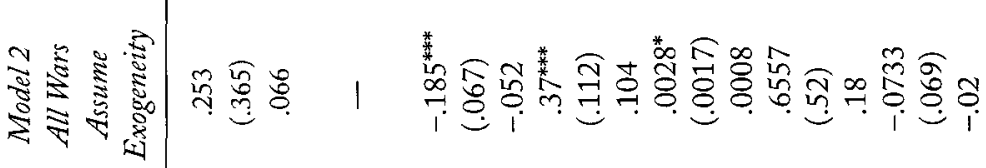

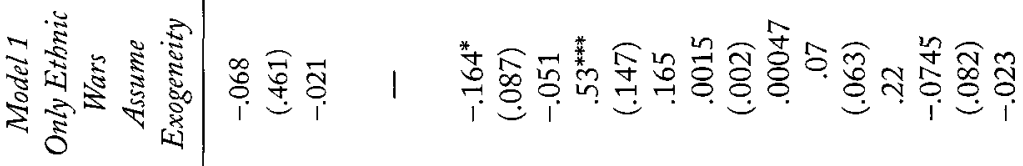

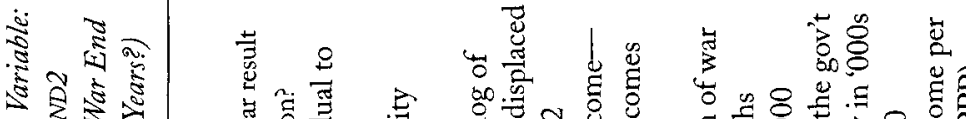

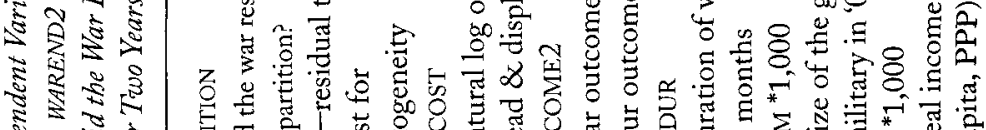

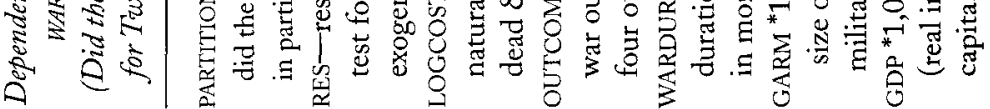




$$
\begin{aligned}
& \text { || }
\end{aligned}
$$

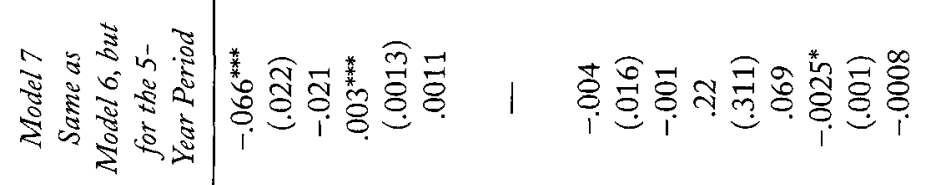

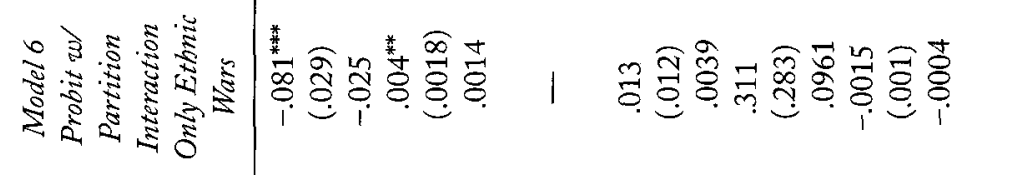

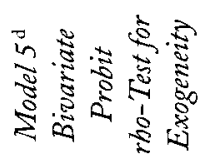

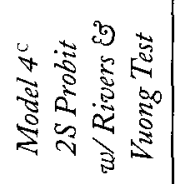

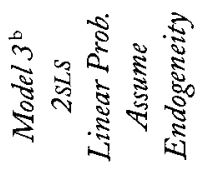

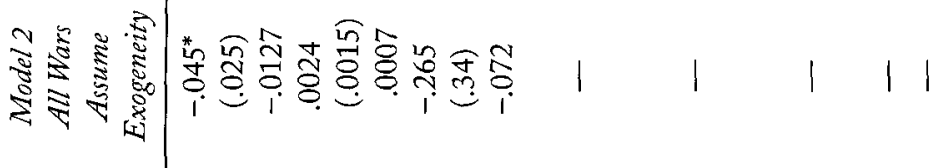

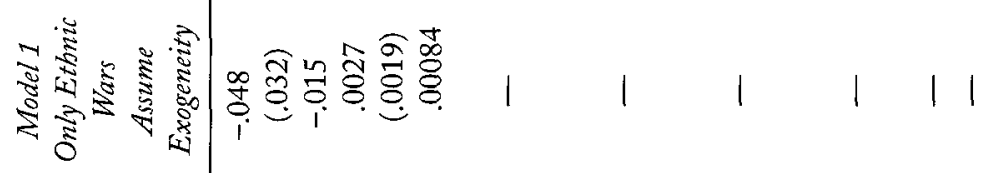

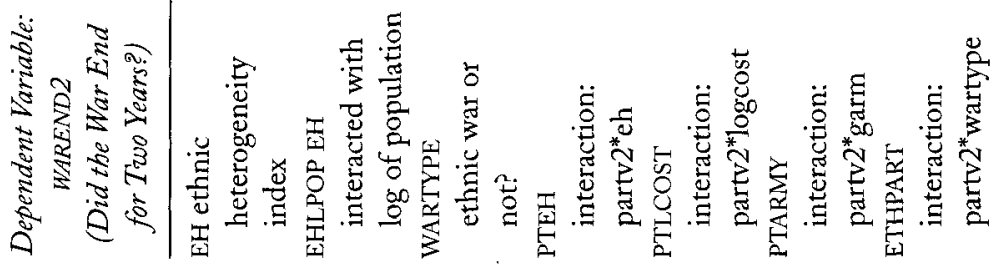




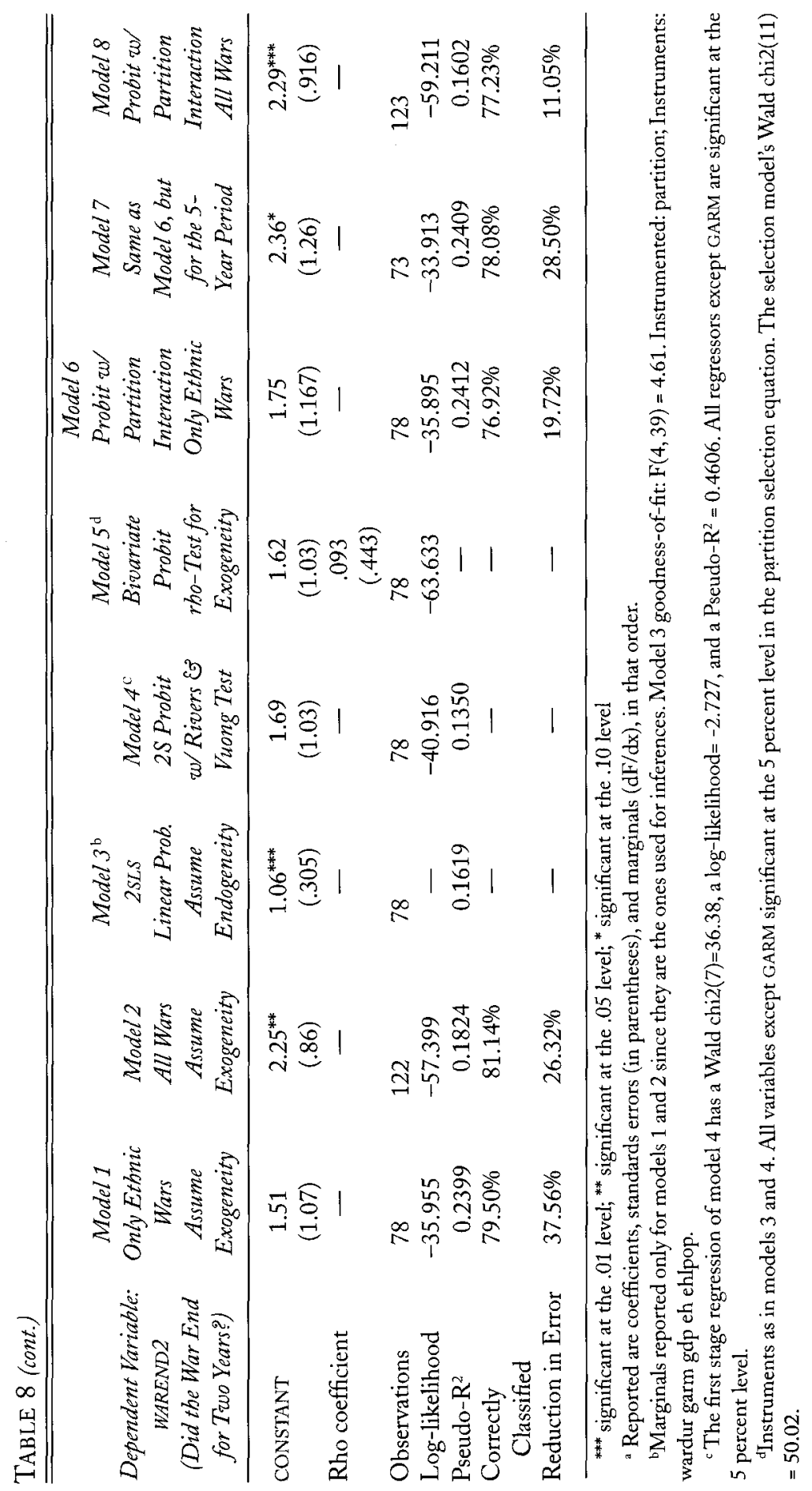


the basis of previous research on war termination. A regressor is used only if at least one other scholar has identified it as significant for war termination and peace building. ${ }^{65}$

The results of models 1 and 2 of Table 8 are quite robust to specification tests. A first interesting finding is that, though not significant in either model, partition is positively correlated with ethnic war recurrence in model 1 (it is negatively correlated with WAREND). ${ }^{66} \mathrm{It}$ is, however, positively correlated with WAREND if we look at the entire population of wars. In fact, among ideological nonethnic wars, partition is a significant determinant of war termination, which implies that nonethnic partitions are more stable and peaceful than ethnic partitions (although these results are driven by the very few cases of nonethnic partition). ${ }^{67}$

The human cost of the war in both models 1 and 2 is negatively correlated with war ending, confirming that the greater the number of people injured by the war, the harder it is to build peace. ${ }^{68}$ War outcomes are the most significant and robust variable, though the OUTCOME 2 variable is hard to interpret (Table 9 below explains these effects more clearly). ${ }^{69}$

${ }^{65}$ Following are the explanatory variables and the researchers who identified their importance: war duration, Mason and Fett (fn. 33); size of the government's military, Mason and Fett (fn. 33); war outcomes, Licklider (fn. 32) and Walter (fn. 39); ethnic heterogeneity and population size, Collier and Hoeffler (fn. 45); deaths and displacements, Licklider (fn. 32) and Doyle and Sambanis (fn. 45); income per capita, Collier (fn. 45); major power involvement, Singer and Small (fn. 32); foreign intervention, Regan (fn. 33); third-party and UN peace operations, Doyle and Sambanis (fn. 45); democracy, Collier, E1badawi, and Sambanis (fn. 30) and Håvard Hegre et al., "Towards a Democratic Civil Peace? Opportunity, Grievance, and Civil War, 1816-1992" (Paper presented at the World Bank Conference on the Economics of Political and Criminal Violence, Washington, D.C., February 16-22, 1999). I also controlled for the number of land borders, the decade the war started, and the cold war. I could not include too many of these variables together, given my small data set and the collinearity of these variables.

${ }_{66}$ The Russia-Chechnya case causes the negative sign, and partition is positively correlated with WAREND if I drop that observation. The nonsignificance of partition, however, does not change by deleting that observation. I also sequentially deleted several other cases (e.g., Cyprus 1963/67, Somalia, Tajikistan, India), and the substantive results did not change. The results are also robust to using TYPELICK and TYPESTF instead of WARTYPE to identify ethnic wars.

67 The opposite argument (without much empirical support) is made in Chaim Kaufmann, "Intervention in Ethnic and Ideological Civil Wars: Why One Can Be Done and the Other Can't," Security Studies 6 (Autumn 1997).

${ }^{68}$ The sign of LOGCOST in models 1 and 2 is negative, indicating that the higher the human cost, the greater the probability of war recurrence. This may seem counterintuitive: why would more costly wars lead to new wars? Would not great human cost discourage war recurrence? That reasoning is correct and it is reflected in my findings on war duration (see below), which verify the war-weariness hypothesis. However, controlling for this finding, the probability of war recurrence should be expected to be greater as the human costs of the war increase. These costs measure war-generated hostility and create grievances that may manifest themselves in future conflict. Further, the greater the human and economic cost of the war, the lower should be a country's human capital and the lower the state's capacity to resume normal operations.

${ }^{69}$ OUTCOME2 is a categorical variable denoting whether the war ended in a truce, rebel victory, government victory, or peace settlement. It is highly significant in both models 1 and 2, though interpreting its sign is not straightforward. By disaggregating OUTCOME2, I found some of its components highly correlated with partition. Thus, entering them independently in the regression would increase collinearities. These correlations also generate sufficient concern over the possible endogeneity of partition when both OUTCOME2 and partition are included in the model. 
TABLE 9

First Differences of the Probability of War Recurrence FOR ETHNIC AND ALL CIVIL WARS ${ }^{a}$

\begin{tabular}{|c|c|c|c|c|}
\hline \multirow{2}{*}{$\begin{array}{l}\text { Only Ethnic Wars } \\
\text { Difference of } \\
\text { PARTITION } \\
\text { from } 0 \text { to } 1\end{array}$} & \multirow{2}{*}{$\begin{array}{c}\text { Estimate of Mean } \\
\text { Change in } \\
\text { Prob }(\text { warend } 2)=1 \\
-.027\end{array}$} & \multirow{2}{*}{$\begin{array}{c}\begin{array}{c}\text { Standard } \\
\text { Deviation }\end{array} \\
\text { of Estimate } \\
.174\end{array}$} & \multicolumn{2}{|c|}{$\begin{array}{c}95 \% \\
\text { Confidence } \\
\text { Interval }\end{array}$} \\
\hline & & & -.374219 & .3025837 \\
\hline $\begin{array}{l}\mathrm{EH} \\
\text { from } 25^{\text {th }} \text { to } \\
75^{\text {th }} \text { percentile }\end{array}$ & -.601 & .322 & -.9731405 & .2372383 \\
\hline $\begin{array}{l}\text { EHLPOP \& EH } \\
\text { from } 25^{\text {th }} \text { to } \\
75^{\text {th }} \text { percentile }\end{array}$ & .002 & .095 & -.1844085 & .1865729 \\
\hline $\begin{array}{l}\text { OUTCOME } 2 \\
\text { from truce to treaty }\end{array}$ & .119 & .027 & .064833 & .1706 \\
\hline $\begin{array}{l}\text { LOGCOST } \\
\text { from } 25^{\text {th }} \text { to } \\
75^{\text {th }} \text { percentile }\end{array}$ & -.182 & .097 & -.3737424 & .0045121 \\
\hline
\end{tabular}

\begin{tabular}{|c|c|c|c|c|}
\hline \multirow{2}{*}{$\begin{array}{l}\text { All Civil Wars } \\
\text { First Difference of } \\
\text { PARTITION } \\
\quad \text { from } 0 \text { to } 1\end{array}$} & \multirow{2}{*}{$\begin{array}{c}\begin{array}{c}\text { Estimate of Mean } \\
\text { Change in } \\
\text { Prob }(\text { warend2 })=1\end{array} \\
.088\end{array}$} & \multirow{2}{*}{$\begin{array}{c}\begin{array}{c}\text { Standard } \\
\text { Deviation } \\
\text { of Estimate }\end{array} \\
.129\end{array}$} & \multicolumn{2}{|c|}{$\begin{array}{c}95 \% \\
\text { Confidence } \\
\text { Interval }\end{array}$} \\
\hline & & & -.1755012 & .3330738 \\
\hline $\begin{array}{l}\text { EH } \\
\text { from } 25^{\text {th }} \text { to } \\
75^{\text {th }} \text { percentile }\end{array}$ & -.621 & .269 & -.9458801 & .1065577 \\
\hline $\begin{array}{l}\text { EHLPOP \& EH } \\
\text { from } 25^{\text {th }} \text { to } \\
75^{\text {th }} \text { percentile }\end{array}$ & -.082 & .067 & -.2188984 & .0452573 \\
\hline $\begin{array}{l}\text { OUTCOME } 2 \\
\text { from truce to treaty }\end{array}$ & .098 & .025 & .0507963 & .1499487 \\
\hline $\begin{array}{l}\text { LOGCOST } \\
\text { from } 25^{\text {th }} \text { to } \\
75^{\text {th }} \text { percentile }\end{array}$ & -.246 & .088 & -.416339 & -.0723445 \\
\hline $\begin{array}{l}\text { WARTYPE } \\
\text { from ethnic to } \\
\text { ideological }\end{array}$ & -.094 & .123 & -.3369971 & .1517012 \\
\hline
\end{tabular}

${ }^{a}$ Changes in $\mathrm{X}$-variables are noted in the first column. All other variables are held constant at their means. Some of these probability changes are not statistically significant, as can also be seen in Table 8 .

Generally, the more conciliatory the outcome of the war, the greater the probability that the peace will last. War duration is positively correlated with no war recurrence, but it is only significant in model 2 . This result is consistent with the findings of Mason and Fett and weakly supports 
the "war weariness" hypothesis. ${ }^{70}$ The size of the government's military is also positive but nonsignificant (it is significant only at the 10 percent level in model 2). ${ }^{71}$ Real GDP per capita is negatively correlated with war ending, though this association is not significant. ${ }^{72}$

The impact of ethnic heterogeneity on war recurrence is quite interesting. Ethnic heterogeneity $(\mathrm{EH})$ and its interaction term with the log of population (EHLPOP) are not significant in models 1 and 2, despite the emphasis on ethnicity in the civil war literature. Moreover, EHLPOP has a positive sign, indicating that if the ethnic groups are large enough to protect themselves against domination, the risk of war recurrence is smaller. This result is consistent with Fearon and Laitin's analysis of the potential for interethnic cooperation and is robust to different measures of ethnic division (the ELF index) and different model specifications. ${ }^{73}$ As I added several interaction terms between partition and other variables in models 6-8, I found a significant negative association between ethnicity and lasting peace but also a significant positive association between peace and larger ethnic groups. Other authors have found that ethnolinguistic and religious fractionalization is a significant determinant of the initiation of civil wars, but my findings suggest that civil war initiation and war recurrence are different phenomena with respect to the role of ethnicity and that ethnic diversity is not as detrimental to peace as many tend to assume. ${ }^{74}$

Models 1 and 2 present short-term results. To see if these results also hold in the medium term, I reestimated models 1 and 2 both five and ten years after the end of the war: the model's fit was roughly the same. However, while no major differences occur in the five-year model, in the ten-year model, war duration is no longer significant and partition is highly significant and negatively correlated with war termination. ${ }^{75}$ In models 6-8 I added several interaction terms between partition and LOGCOST, WARTYPE, EH, and GARM, but no appreciably important associations emerged. In model 8 the coefficient of ETHPART-denoting

\footnotetext{
${ }^{70}$ Mason and Fett (fn. 33). I thank Russ Leng for pointing this out to me.

${ }^{71}$ By contrast, Mason and Fett (fn. 33) find a significant negative correlation.

${ }^{72}$ This finding may be due to selection effects, as I mentioned earlier, but it may also confirm the revenue-seeking economic model of civil war in Collier (fn. 45); and Collier and Hoeffler (fn. 45); the model looks at GDP per capita as a proxy for "lootable" resources, which would increase the risk of war.

${ }^{73}$ James Fearon and David Laitin, "Explaining Interethnic Cooperation," American Political Science Review 90 (December 1996).

${ }^{74}$ See, e.g., Collier, Elbadawi, and Sambanis (fn. 30).

${ }^{75}$ This could be due to missing observations. The ten-year model was estimated on fifty cases. The coefficient and robust standard error of partition were -1.137 and .627 , respectively, yielding a $\mathrm{z}^{-}$statistic of $-1.813(\mathrm{P}>|\mathrm{z}|=0.070)$ and a model $\log$-likelihood $=-23.460$, with a Wald chi2 $(8)=32.14$ and a Pseudo $\mathrm{R}^{2}=0.3199$
} 
ethnic partition-is negatively but not significantly correlated with war termination.

These findings build a strong case against partition theory. Before accepting them, I should address a methodological point with potentially substantive implications. My inferences may depend on the assumption that partition is exogenous to war recurrence, an assumption that makes sense, since war recurrence is observed after the occurrence of partition. It may be, however, that these two variables are jointly determined by a common set of explanatory variables. Thus, there is concern over the possible endogeneity of partition. I therefore estimated several models assuming partition is endogenous and I also tested its exogeneity (see the appendix for technical details and for an explanation of the statistical concept of endogeneity). I present the results of three models that address the issue of endogeneity in Table 8.

I estimate a parsimonious linear probability model treating partition as an endogenous variable (model 3 ). The model uses several variables that are not correlated with war recurrence (based on the previous estimations) as instruments for partition and reduces the number of exogenous variables in the structural equation to reduce noise in the system of equations. The model has good fit and is well specified. Despite this estimation adjustment, however, partition is not at all significant and continues to have a negative sign. The other key variables (LOGCOST, OUTCOME2, GDP) behave in much the same way as they did in the previous models.

In models 4 and 5 I formally tested (and could not reject) the null hypothesis that partition is exogenous. Model 4 is a two-stage probit model, estimated following Madalla and using the Rivers and Vuong exogeneity test (which was originally designed for continuous endogenous explanatory variables). ${ }^{76}$ The exogeneity test consists of a t-test of the estimated coefficient of the predicted residual (RES) of the first-

${ }^{76}$ G. S. Maddala, Limited Dependent and Qualitative Variables in Econometrics (Cambridge: Cambridge University Press, 1983); D. Rivers and Q. Vuong, "Limited Information Estimators and Exogeneity Tests for Simultancous Probit Models," Journal of Econometrics 39 (1988). This method is almost identical to Kenneth A. Bollen, D. K. Guilkey, and T. A. Mroz, "Binary Outcomes and Endogenous Explanatory Variables: Tests and Solutions with an Application to the Demand for Contraceptive Use in Tunisia," Demography 32 (February 1995). The two-stage probit model produces inefficient standard errors, though the efficiency loss is small. Rivers and Vuong derive the formula that gives the correct variance-covariance matrix, but their procedure is designed for continuous endogenous right-hand-side variables. A methodological discussion and Monte Carlo simulation results reporting the properties of this estimator in small samples are found in Michael Alvarez and Jennifer Glascow, "Two-Stage Estimation of Non-Recursive Choice Models," Political Analysis (forthcoming). For a political science application of this method, see Michael Alvarez and L. Butterfield, "The Resurgence of Nativism in California? The Case of Proposition 187 and Illegal Immigration," Social Science Quarterly (forthcoming). A discussion of the two binary variable case can be found in Madalla (p. 246). 
stage reduced form equation of partition. The estimated coefficient of RES is .122 and its standard error is .956, so we cannot reject the null hypothesis that partition is exogenous. To confirm this result, I estimated model 5-a bivariate probit model with sample selection (which is a full-information maximum likelihood model)-using the same instruments for partition and the same exogenous variables as in models 3 and $4 .{ }^{77}$ The correlation coefficient rbo (see Table 8) is .093 and its standard error is .443. A Wald test of the hypothesis that $r b o=0$ yields chi2 $(1)=.043$, which does not allow us to reject the exogeneity of partitions at the 0.8346 level. Thus, more efficient estimates are obtained from models 1 and 2 and we can rely on the previously discussed inferences.

Table 9 provides some easily interpretable results of the estimated change in the probability that there will be no war recurrence as a result of changes in key explanatory variables (these changes are also known as first differences). I reestimated models 1 and 2 from Table 8 and simulated (with one thousand repetitions) their parameter estimates using the CLARIFY software. ${ }^{78}$ Using those estimates, I obtained the first differences reported. ${ }^{79}$ I report the mean and standard deviation of the estimated change in probability (the standard deviation reflects the significance levels of the explanatory variables as in Table 8). Note that the probability of no war recurrence (peace) is slightly reduced by a partition after an ethnic war, although it is increased by 8.8 percent when we use the entire population of civil wars (though this result is not statistically significant). Among the statistically significant effects, it is worth reporting that if the war ends in a treaty instead of a truce, the probability of no war recurrence increases by 11.9 percent in ethnic wars and 9.8 percent in all wars. Finally, the more costly the war, the less stable the peace. By varying LOGCOST from its 25 th to its 75 th percentile, the probability of no war recurrence drops by 18.2 percent in ethnic wars and 24.6 percent in all wars.

To conclude this section, the evidence does not support the assertion that partition significantly reduces the risk of war recurrence. Hence

\footnotetext{
${ }^{77}$ For the case of two binary variables, this can be estimated as a seemingly unrelated bivariate probit model with a selection effect. The exogeneity test in this model consists of a Wald test of $r b o$, the estimated coefficient of the correlation of the error terms in the structural and reduced-form ("firststage") equations.

${ }^{78}$ Michael Tomz, Jason Wittenberg, and Gary King, "Clarify: Software for Interpreting and Presenting Statistical Results," version 1.2.1 (June 1, 1999). See also idem, "Making the Most of Statistical Analyses: Improving Interpretations and Presentation" (Paper presented at the annual meeting of the American Political Science Association, Boston, 1999).

${ }^{79}$ Note that these estimates may differ slightly in replications since I did not fix the number seed used to randomly select samples for the simulations.
} 
there is no support for partition as a policy option if the rationale advanced is that it will prevent future ethnic wars.

\section{Does Partition End Ethnic Violence Short of War?}

The third critical hypothesis in support of partition is that the physical separation of ethnic groups will reduce residual, low-level ethnic violence (that is, violence short of war). In this section I test this hypothesis empirically and find that only under carefully specified conditions does partition reduce lower-level violence. In most situations partition will have a negligible effect on residual violence.

The dependent variable in the models estimated in this section is NOVIOL, which takes the suffixes 2 and 5 when it is measured two and five years after the end of the war, respectively. NOVIOL is binary and it is coded 1 if there is no residual violence after the end of the war and 0 otherwise. It is coded based on available information and other data sets that code armed conflict short of war and/or politicides and genocides (a table with details on the coding of each case is included in the online data set). I use two versions of the dependent variable, NOVIOL 2 and NOVIOL5, for two and five years after the end of the war.

I start by regressing NOVIOL 2 on the explanatory variables from the war-recurrence model, which does not produce many significant results and has low explanatory power. Partition is positively but nonsignificantly associated with an end to lower-level violence (model 1 in Table 10 ). Given the model's low classification success, it appears that war recurrence and residual violence are substantially different phenomena, so I changed the model's specification in regressions 2-6. First, I disaggregated the OUTCOME 2 variable and focused on the signing of treaties, which in model 2 increased the significance level of partitions by doubling its coefficient and reducing its standard error. Treaty is also significant and positive, suggesting that treaties generally do signal the parties' intentions to reconcile their differences. We also find that EHLPOP is significant and positive. ${ }^{80}$ Both results seem important. The

\footnotetext{
${ }^{80}$ This result complements Bates's (fn. 47) findings on the relationship between ethnicity and political violence in Africa. Bates studies the relationship between ethnicity and economic modernizationurbanization, education, and the rise of per capita income, as well as political participation. Focusing on forty-six African countries from 1970 to 1995, he tests the relationship between ethnicity and political violence at several levels (not just wars). He measures the size distribution of ethnic groups, linguistic diversity, and the presence of an ethnic minority at risk, based on the work of a number of other researchers, and shows that the relationship between ethnicity and violence is complicated. Bates finds that "controlling for the impact of other variables, [linguistic diversity] associates with higher levels of violence ... the size of the largest ethnic group enters quadratically; when the coefficient for the linear term is significant, so too is the coefficient for the quadratic. But as the size of the largest ethnic group grows, the level of violence initially decreases, but then increases; by contrast, the level of protest
} 
first result seems to support partition theory, though the latter seems to contradict it. These results are essentially the same for the five-year period (NovioL5; see model 3 in Table 10). ${ }^{81}$

Models 3 and 4 are differently specified to facilitate sensitivity analysis of the previous results and to achieve higher classification success. Model 3-discussed previously-applies to the five-year period and has more than double the classification success of the two-year model. It shows that partition is not significant for ending residual violence. In model $4 \mathrm{I}$ added the variable INTENSE, which measures the war's intensity and which is positive and extremely significant. Very intense wars seem either to provide no further appetite for violence or to eradicate all resistance early on, so no residual violence is necessary. The GARM variable is not significant in any of the regressions and neither is economic development (proxied by electricity consumption per capita). ${ }^{82}$ In model 4 we find that residual violence is significantly reduced by long and bloody wars, but again partition is not significant. Further, the partition variable is extremely fragile to small specification changes. Contrast models 4 and 5, which differ only in that model 4 controls for LOGCOST and model 5 for LOGDEAD (that is, not for displaced persons). The coefficient of the partition variable drops from .67 to .27 (the marginal effect drops from .26 to .10). The other variables are much more stable.

Model 6 is the best-performing model specification; it controls for civil wars in the same country during the previous ten years. That variable is extremely significant and suggests that residual violence is much more likely if the country has had a history of civil war recurrence. This effect is mitigated, however, in the case of long and intense wars, which discourage residual violence. Model 6 has substantially higher classification success than the previous models, but, in general, the models of

initially increases, but then falls" (p. 25). In Bates's analysis, it is extreme polarization that is most associated with violence. My finding that greater heterogeneity and more sizable ethnic groups reduce violence in postwar states is therefore compatible with Bates's result and complements it. I should note however, that Bates's results may not be generally applicable to non-African countries, given that African countries have a generally higher mean level of ethnic heterogeneity and this implies a selection effect if the results are to be applied widely to non-African countries. In my data set, for example, the mean and standard deviation of the ethnic heterogeneity index for African countries is 68.82 and 34.58 , respectively, whereas for non-African countries it is 50.5 and 31.73 . The ethnic heterogeneity index used was created by Tatu Vanhanen, "Domestic Ethnic Conflict and Ethnic Nepotism: A Comparative Analysis," Journal of Peace Research 36, no. 1 ( 1999).

${ }^{81}$ Ten years after the end of the war partition has a negative correlation with an end to low-grade violence, but this result may be an artifact of missing (right-censored) observations (only fifty-five observations are available for the ten-year period).

${ }_{82}$ Thus, development levels and military strength are more relevant with respect to war recurrence than with respect to low-level violence. 


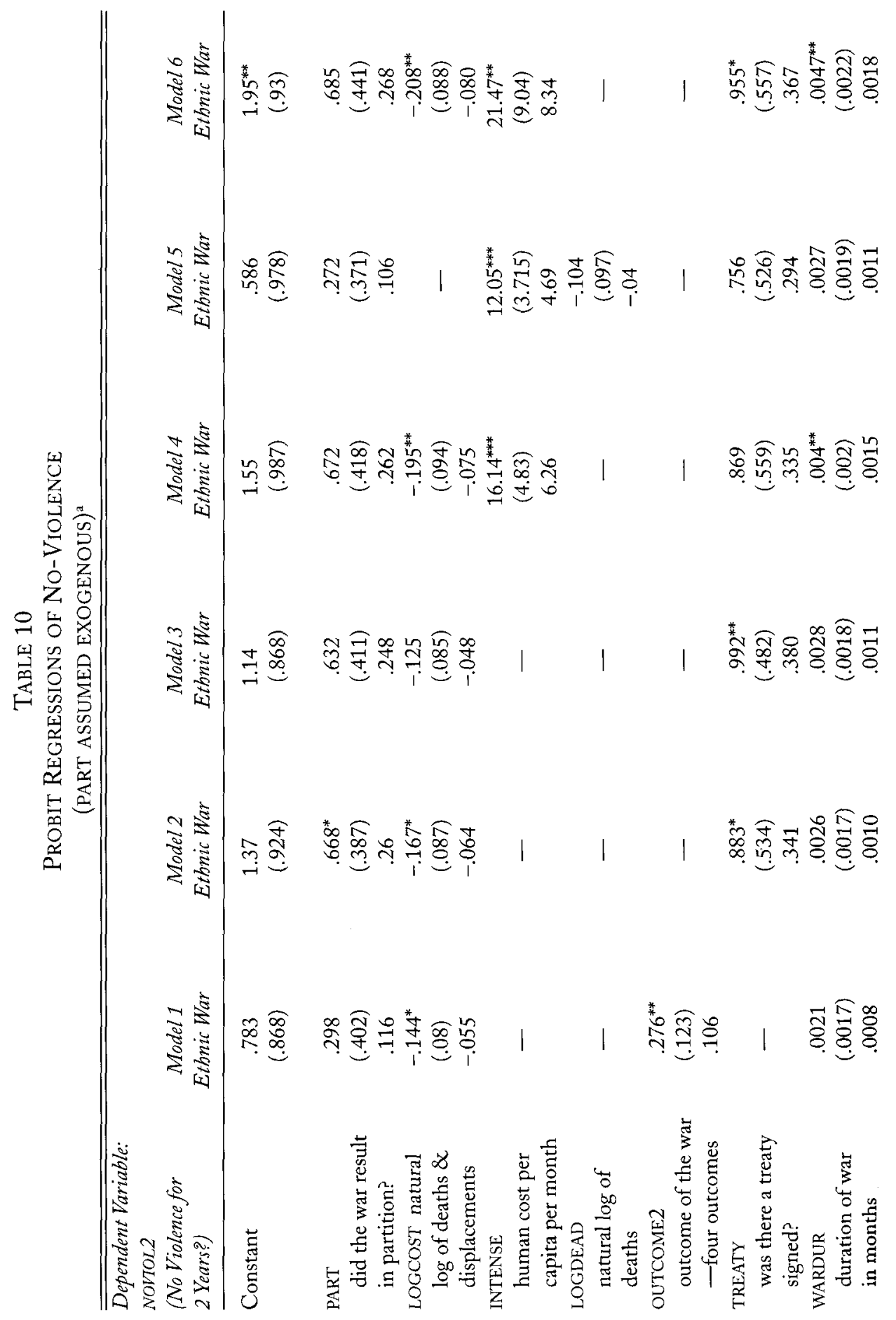




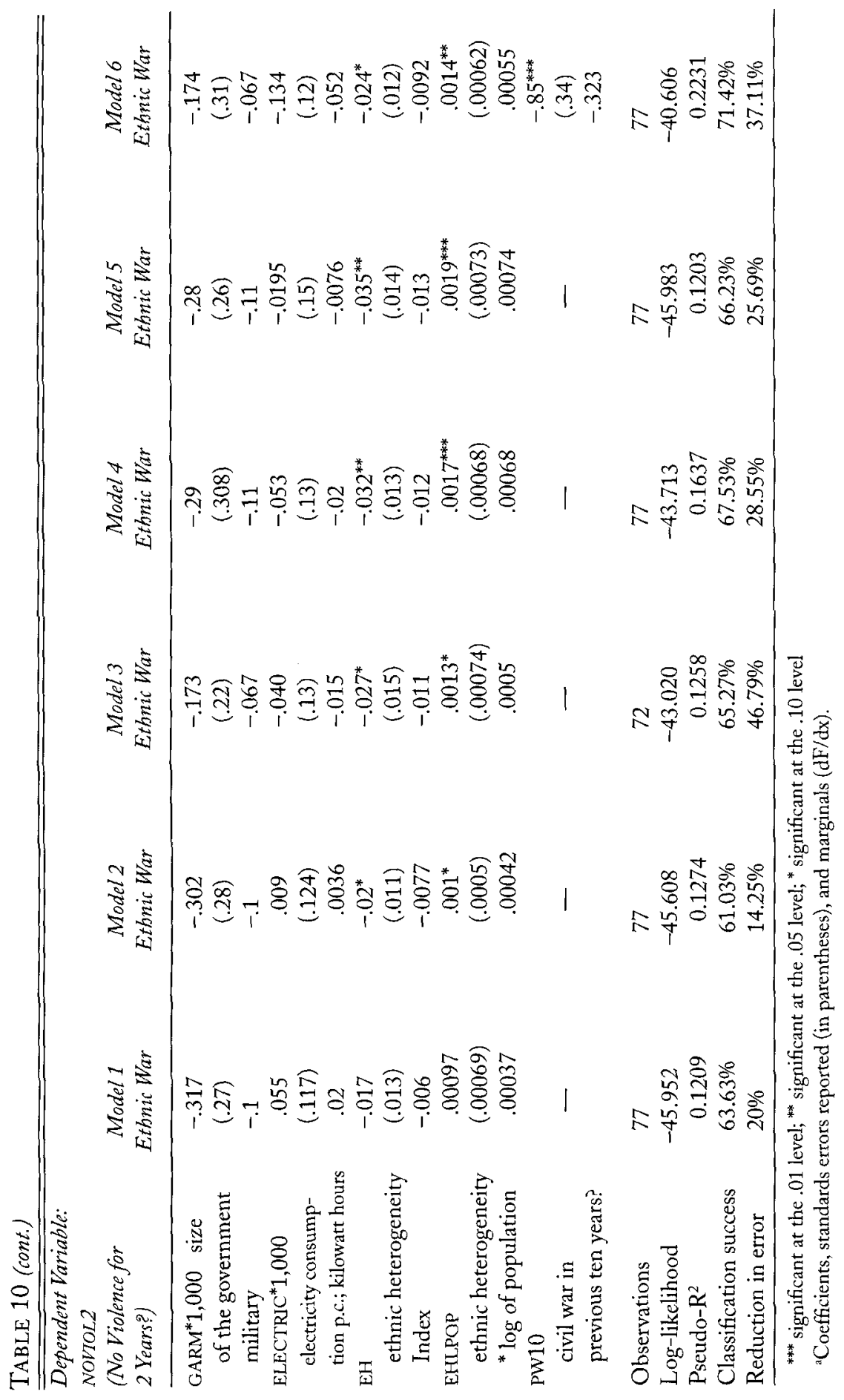


residual violence do not fit the data as well as the models of war recurrence discussed earlier. Thus, the lack of significance of the partition variable may be a function of omitted variables that could be identified in further theoretical research. At the same time model 6 confirms the results discussed earlier with respect to the positive impact of ethnic diversity, if ethnic groups are large (EHLPOP variable).

In sum, I can point to only very weak evidence in support of the hypothesis that partitions help end low-level ethnic violence (in model 2 and some variants). More importantly, the positive impact of partitions seems fragile and extremely dependent on whether or not the war ended in a treaty, on the war's intensity, on the number of people displaced by the war, and on the number and size of ethnic groups.

To confirm that these results are not influenced by the potential endogeneity of partitions to low-grade violence, I tested once again for exogeneity. I estimated model 4 using a 2 sLs linear probability model using many different combinations of instrumental variables to see whether the coefficient of partition would become significant if endogeneity is assumed. I used as instruments only variables that were not significantly associated with low-level violence in the previous regressions. I could not, however, find any results that would increase my confidence in the significance of the partition variable. ${ }^{83}$

To summarize, I find only weak support for the hypothesis that partitions are significant for an end to low-grade ethnic violence after civil war. Models supporting that hypothesis are very sensitive to small specification changes. Models controlling for the potential endogeneity of partition are not robust, have poor fit to the data, and are quite sensitive to the choice of instrumental variables.

\section{CONCLUSION}

Population movements to partition states during or after civil war are coerced, painful, and costly, and they may sow the seeds of future conflict. It is therefore imperative that international policy toward partition

\footnotetext{
${ }^{83}$ Results are available from the author. Finding good instruments in this data set has proven notoriously difficult. I did not change the specification of model 4; rather I just added and dropped instruments and considered other variables as potentially endogenous (specifically, WARDUR, LOGCOST, TREATY, ENERCAP). The instruments I used in various combinations were GEO, BOR1DER, EH, GDP, URBST (urban population at the start of the conflict), GARM, INTERVEN (was there an external intervention?), MAJOR (was there a major power involved?). I found only one permutation that made partition significant, but when I estimated this model using a bivariate probit estimator, I found that the error terms of the structural and reduced-form first equation were perfectly correlated ( $r b o=1$ ), which indicates either that there was too much noise in the system or that the distribution is not a bivariate normal.
} 
be informed by rigorous, empirically verified arguments, rather than by untested theory. In this paper, I have provided a host of empirical tests, starting with an empirical inquiry into the determinants of war-related partition. I have found that partitions are more likely after costly ethnic/religious wars, after rebel victory or truce, and in countries with better-than-average socioeconomic conditions. Partitions are more likely where ethnic groups are large; they are less likely to occur as the degree of ethnic heterogeneity increases.

My analysis has also shown that the differences between ethnic and nonethnic wars with respect to war termination and partition are small. The relationship between the degree of ethnic heterogeneity and the need for partition is not as straightforward as partition theorists assume. ${ }^{84}$ The finding that partition does not significantly prevent war recurrence suggests, at the very least, that separating ethnic groups does not resolve the problem of violent ethnic antagonism.

These findings lead me to formulate a new hypothesis for future research: if border redefinition is in the cards after civil war (or before war), then the strategy of supporting ethnic diffusion by combining rather than partitioning large ethnic groups may be worth pursuing. Thinking along these lines could be very useful for Africa in particular, given the persistent concern about the "unnaturalness" of Africa's borders and the recent debate about redefining those borders in the hope of reducing the incidence of civil wars. ${ }^{85}$ Partition theorists would argue for the partition of warring African states into a multitude of ministates each of which is composed of a single ethnicity group. Even if this solution reduces the incidence of internal war, it will almost certainly increase the incidence of international war. Based on the empirical findings of this paper, I would put forth a rival hypothesis: if borders can be credibly and securely redrawn, then combining several large ethnic groups in a larger multiethnic state may reduce the probability of new wars. It would be useful for further research to try to clarify any threshold effects that may be associated with the size of ethnic groups as they affect the likelihood of violent secessionist activity.

No doubt this proposal is difficult, perhaps infeasible. Skeptics, however, should at least acknowledge that the political dangers of ethnic diversity are imperfectly understood and that, despite the practical

\footnotetext{
${ }^{84}$ To cite Horowitz (fn. 2), 135: "Is there any reason to believe that the more pronounced the cultural differences that exist between groups, the greater the ethnic conflict? There has been no shortage of offhanded assertions that cultural differences engender ethnic conflict. But ... systematic statements of the relationship are more difficult to find."

${ }_{85}$ See, e.g., Jeffrey Herbst, "Responding to State Failure in Africa," International Security 21 (Winter 1996-97).
} 
difficulty of my proposal, it is at least based on a more nuanced understanding of the relationship between ethnicity and political violence. Indeed, much of the best recent research on political violence consistently points to the fact that ethnic diversity need not generate violence. Economic studies of the occurrence of civil war in a sample of 161 countries have found a parabolic relationship between ethno-linguistic and religious fractionalization and the onset and duration of civil wars. ${ }^{86}$ Work in political science, too, has steadily reinforced this point. ${ }^{87}$ Bates, for example, casts "doubt upon a . . . tenet of conventional wisdom: that ethnic diversity promotes violence." He finds that the "red zone" for violent conflict occurs "when an ethnic bloc may be sufficient in size to permanently exclude others from the exercise of power." 88 Thus, enhancing ethnic diversity while strengthening political institutions can be beneficial. So why partition states to reduce ethnic diversity?

Perhaps there are other benefits to be derived from partition-democratization, for example. The jury is still out on this question. My empirical analysis shows that partitions may have such an effect, but this effect may be due to the prewar institutions of civil war-torn countries; such institutions may be at least as important as partition in determining the democratic future. And the process of democratization itself may harbor dangers and cause violence. ${ }^{89}$ Thus, international policy aimed at preventing war recurrence should promote institution building and socioeconomic development before war occurs in the first place, rather than supporting partition after war occurs.

Partition, as we have seen, does not help reduce the risk of war recurrence. Partitions are in fact positively (though not significantly) associated with recurrence of ethnic war. The probability of a new war rises in tandem with the human toll of the previous war and with nondecisive outcomes to the war. War recurrence is also positively, though not significantly, associated with GDP per capita ${ }^{90}$ and with ethnic heterogeneity, though as ethnic groups become larger, new wars tend to

${ }^{86}$ Collier, Elbadawi, and Sambanis (fn. 30).

${ }^{87}$ Laitin and Fearon (fn, 73).

${ }^{88}$ My findings need not agree entirely with Bates's (fn. 47), since our samples and research questions differ. I have not measured the size of the largest ethnic group, which is critical in his argument, but I do find that greater ethnic heterogeneity within the context of a larger population reduces the risk of war recurrence and residual violence. This is consistent with Bates.

${ }^{89}$ Bates (fn. 47), 28. The dangers of the process of democratization, as opposed to the end goal of democracy, should not be underestimated. On the potential of regime transitions, including democratic transitions, to create civil war, see Hegre et al. (fn. 65).

${ }_{90}$ This correlation between GDP and war recurrence may seem counterintuitive since we saw that higher GDP is correlated with higher democracy, which is negatively correlated with civil war. The impact of GDP is ambiguous because GDP can be a proxy both for the country's overall development level, which should be positively associated with peace, and for "loot," inciting new wars; see Collier and Hoeffler (fn. 45). 
become less likely. ${ }^{91}$ Negotiated settlements, a strong government army, and a lengthy previous war all reduce the probability of war recurrence. Thus, if the international community's interest lies in preventing new civil wars, it could manipulate some of these significant variables toward desirable goals. It could, for example, take steps to enhance the government's military and support decisive war outcomes. ${ }^{92} \mathrm{Or}$ it could support the negotiation of peace treaties, which reduce the threat of new violence. Again, what the international community should not do to prevent future wars is promote partition.

One benefit that can come from partition is the reduction of residual low-level ethnic violence. Certainly, it follows that if ethnic groups divide into ethnically homogeneous territorial units, the risk of ethnic conflict declines. I have found that this result depends critically on the number of displaced people, as well as on the way the previous war ended and on the country's longer war history. The probability of lowlevel violence is shaped by some of the same variables that influence war recurrence, but it also is a function of different determinants. The previous discussion of how to prevent war therefore applies to residual violence, but with some modifications. To reduce residual violence, it is important to prevent war recurrence, as patterns of large-scale violence over time seem to encourage lower-level violence.

Fine-tuning a war-to-peace transition is a difficult task because peace-building strategies can often backfire. For example, promoting economic growth may assist democratization and promote peace, but it can also lead to new wars by expanding the potential economic gains from a new rebellion. Strategies to support the government's prewar institutions and its military may also achieve peace, but they may do so at the expense of justice.

Muddling through this difficult terrain, I would propose an empirically derived strategy for resolving ethnic wars. This strategy demands action by the international community, which must promote democracy as its number one conflict-prevention strategy. If violence does erupt, its priority should be to facilitate a negotiated settlement, as well as to integrate and downsize the government's military. According to my empirical analysis, these strategies can be effective. If border redefinition is a viable option-and it should be an option only if it does not

\footnotetext{
${ }^{91}$ Despite the fact that this study looks only at cases where war has already taken place, the results on the impact of ethnic heterogeneity are compatible with studies of initial war occurrence, in that ethnic heterogeneity seems to increase the risk of war at first, but as the degree of heterogeneity increases, that risk declines. In my analysis, however, this effect has not been statistically significant.

${ }^{92}$ Such a strategy, however, might indirectly support political repression, so it must be carefully and selectively applied and militaries should integrate the rebel army if possible.
} 
assist one party at the expense of another-then ethnic integration rather than ethnic partition may be a winning strategy. In addition to having the potential for greater success than partition, this strategy is also not loaded with subjective and arbitrary assumptions about the necessity for ethnically pure states and about the futility of interethnic cooperation. Only in the most extreme cases may partition be necessary, indeed inevitable. Those cases must be handpicked on the basis of political analysis of regional and global constraints, the history of the preceding war, and the special traits of the society in question. More research on this topic will help pinpoint the benefits and the dangers of partition under different conditions. What this study has suggested is that, on average, partition may be an impossible solution to ethnic civil war.

\section{ApPendix: Testing for THe Possible Endogeneity of Partitions}

A number of explanatory variables are correlated with both partition and no war recurrence. This raises concerns about possible endogeneity of partition in models of war recurrence. This appendix explains the problem of endogeneity to which I refer in the text and explains how the problem can be addressed.

Endogeneity results if a system of simultaneous equations has correlated error terms. Here, we have a system of two equations of partition $(\mathrm{P})$ and war recurrence $(\mathrm{W})$ :

$$
\begin{aligned}
\mathrm{P}_{\mathrm{i}} & =\alpha+\beta_{1} \mathrm{M}_{\mathrm{i}}+\beta_{2} \mathrm{X}_{\mathrm{i}}+\varepsilon_{\mathrm{i}} \\
\mathrm{W}_{\mathrm{i}} & =\gamma+\beta_{3} \mathrm{P}_{\mathrm{i}}+\beta_{4} \mathbf{M}_{\mathrm{i}}+\beta_{5} Z_{\mathrm{i}}+\mu_{\mathrm{i}}
\end{aligned}
$$

where equation 1 determines the incidence of partition $(\mathrm{P})$ and equation 2 determines war recurrence $(W)$. $\mathbf{M}_{i}$ is a vector of common variables in the two equations and $\beta_{1}$ is the vector of coefficients of $\mathbf{M}_{\mathbf{i}} ; \mathbf{X}_{\mathbf{i}}$ is a vector of instrumental variables (correlated with partition but not with war recurrence) and $\beta_{2}$ is a vector of their coefficients; $Z_{i}$ is a vector of variables that are correlated with war recurrence and $\beta_{5}$ is a vector of their coefficients. Endogeneity stems from the possible correlation between $\varepsilon_{\mathrm{i}}$ and $\mu_{\mathrm{i}}$ (both of them disturbance terms with mean zero and no correlation to the other explanatory variables). The presence of common explanatory variables in equations 1 and 2 (that is, the variables in $\mathbf{M}_{\mathrm{i}}$ ) implies that $\varepsilon_{\mathrm{i}}$ and $\mu_{\mathrm{i}}$ may be correlated, in which case, a simple probit regression would produce biased parameter estimates. 
To resolve this problem, we must first test for endogeneity, and if we detect it, we must estimate a model that corrects for it as in the twostage least squares model for linear regression. This problem is harder when the dependent variable is binary. The two-state probit used by Bollen, Guilkey, and Mroz, following Rivers and Vuong, is a model that has been shown to generate consistent parameter estimates with small efficiency loss. This model has attractive properties..$^{93}$ The procedure is similar to 2SLS: first, estimate the reduced form of equation 1 using a probit regression, obtaining predicted values (PHAT) of the dependent variable ( PART) ${ }^{94}$ Then, compute the error of that prediction (RES) and plug the PHAT into equation 2, replacing PART. PHAT is uncorrelated with the disturbance term in equation 1 . Then, estimate equation 2 using a probit regression with robust standard errors. The resulting coefficient estimates are consistent asymptotically inefficient. ${ }^{95}$ However, evidence from small-sample Monte Carlo simulations has shown that the efficiency loss is very small. ${ }^{96}$

To determine whether this process should be used, a simple exogeneity test can be applied. This involves a t-test of the estimated coefficient of the prediction error ( RES), added as a regressor along with the actual value of PART in equation 2 in the model of war recurrence. ${ }^{97}$ If RES is nonsignificant, we cannot reject the null hypothesis of exogeneity of partitions and we should rely on the simple probit estimates. Applying this method to the no-war-recurrence model, I find that more efficient results are reached by models that assume exogeneity. This is confirmed by estimating a bivariate probit model of war recurrence that provides efficient standard errors (estimated by maximum likelihood) and a different test of exogeneity.

\footnotetext{
${ }^{93}$ The choice of estimator depends on the number of observations, the degree of identification of the model, the number of potentially endogenous variables, and the goodness of fit of the first-stage equation. See Bollen, Guilkey, and Mroz (fn. 76).

${ }^{94}$ Use of this method is strictly based on attaining an $R^{2}$ no smaller than 10 percent in equation 1. In my case, the $\mathrm{R}^{2}$ was higher than 30 percent. Further, Bollen, Guilkey and Mroz (fn. 76) discuss evidence from Monte Carlo simulations that suggest that the models' identification must be less than 75 percent for the two-step probit estimator to be preferable to the simple probit (i.e., the overlapping variables in the two equations must be fewer than three-fourths of the total number of right-handside variables in the structural equation).

${ }^{95}$ T. Amemiya, "The Maximum Likelihood and the Nonlinear Three-Stage Least Squares Estimator in the General Nonlinear Simultaneous Equation Model," Econometrica 45 (1978).

${ }^{96}$ See Bollen, Guilkey, and Mroz (fn. 76); and G. Tauchen, "Diagnostic Testing and Evaluation of Maximum Likelihood Models," Journal of Econometrics 30 (1985). Formulas to compute efficient standard errors can be found in Maddala (fn. 76), and a method to estimate the asymptotically efficient covariance matrix when the model is overidentified has been developed by Amemiya (fn. 95). Alvarez and Butterfield (fn. 76) use bootstrapping to obtain estimates of the correct standard errors.

${ }^{97}$ Bollen, Guilkey and Mroz (fn. 76) find this to be the best-performing exogeneity test.
} 